Supporting Information

\title{
Amine-Catalyzed Cascade Reactions of Unprotected Aldoses - An Operationally Simple Access to Defined Configured Stereotetrads or Stereopentads
}

\author{
C. Richter, M. Krumrey, M. Bahri, S. Trunschke, R. Mahrwald \\ Department of Chemistry, Humboldt-Universität zu Berlin, Brook-Taylor Str. 2, 12489 Berlin, \\ Germany \\ rainer.mahrwald@rz.hu-berlin.de
}




\section{Contents}

1. Experimental Procedures 3

2. Optimization of reaction conditions 4

3. Characterization of Products 5

4. Structure determination of initial experiment (Scheme 2) 16

5. Proof of configuration 18

6. Proof of acyl migration 20

7. Proof of configuration of D-manno-22 22

8. Proof of configuration of D-deoxygalacto-25 25

9. Copies of NMR Spectra 29 


\section{Experimental Procedures}

${ }^{1} \mathrm{H},{ }^{13} \mathrm{C}$ NMR and correlation experiments were carried out at $500 \mathrm{MHz}$ and $125 \mathrm{MHz}, 400$ $\mathrm{MHz}$ and $100 \mathrm{MHz}$ and $300 \mathrm{MHz}$ and $75 \mathrm{MHz}$. Chemical shifts are referenced to the residual solvent signal and are given in ppm ( $\delta$-scale). Coupling constants $(J)$ are given in $\mathrm{Hz}$. Data are reported as follows: chemical shift (multiplicity [singlet (s), doublet (d), triplet (t), quartet (q) and multiplet $(\mathrm{m})$ ], coupling constants, integration). Purification of products was accomplished by flash chromatography using silica (Merck silica gel 60, particle size 0.04 $0.063 \mathrm{~mm}$ ) with a solvent mixture of $\mathrm{CH}_{2} \mathrm{Cl}_{2} / \mathrm{MeOH}: 8 / 2$-> $9 / 1$ and hexane / acetone 7:3 -> 8:2. Substances were purchased from Sigma-Aldrich, TCI Europe and ACROS and were used without further purification.

\section{General procedure}

To a solution of aldose $(1 \mathrm{mmol})$ in $2.0 \mathrm{~mL}$ of water, acetyl acetone $(2 \mathrm{mmol}, 200 \mathrm{mg})$ and amine $(0.2 \mathrm{mmol}, 20 \mathrm{~mol} \%)$ were added. After stirring for $24 \mathrm{~h}$, volatile compounds were removed under reduced pressure and the crude mixture purified by column chromatographie $\left(\mathrm{CH}_{2} \mathrm{Cl}_{2} / \mathrm{MeOH}: 8 / 2\right)$. The isolated products were dissolved in pyridine (10 eq. for aldoses and 12 eq for hexoses). To this solution acetic anhydride were added dropwise (10 eq. for aldoses and 12 eq. for hexoses). After stirring for $24 \mathrm{~h}, 50.0 \mathrm{~mL} \mathrm{NaHCO}$ were added and the aqueous phase was extracted three times with $50.0 \mathrm{~mL}$ ethyl acetate. The combined organic phases were washed with $50.0 \mathrm{~mL} \mathrm{NH}_{4} \mathrm{Cl}$, dried over magnesium sulphate, filtrated and the solvent was removed under reduced pressure. The residue was purified by column chromatography on silica (hexane / acetone: $8 / 2$ ). 


\section{Optimization of reaction conditions}

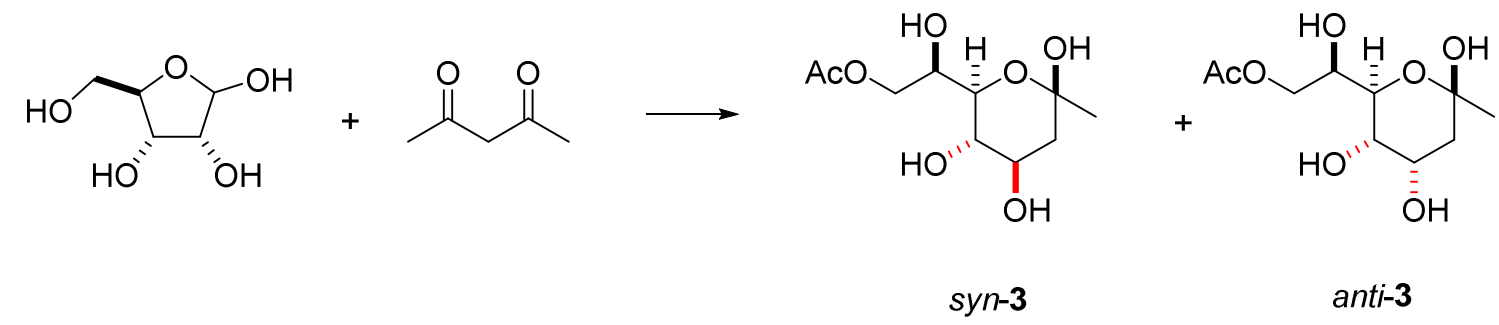

\begin{tabular}{|c|c|c|}
\hline solvent & base & overall yield $^{a}$ \\
\hline $\mathrm{MeOH}$ & Pyrrolidine $(20 \%)$ & $-\%$ \\
\hline DMSO & Pyrrolidine $(20 \%)$ & $-\%$ \\
\hline DMF & Pyrrolidine (20 \%) & $-\%$ \\
\hline $\mathrm{H}_{2} \mathrm{O}$ & Pyrrolidine (20 \%) & $10 \%$ \\
\hline $\mathrm{H}_{2} \mathrm{O}$ & Diisopropylethylenediamine (20 \%) & $40 \%$ \\
\hline $\mathrm{H}_{2} \mathrm{O}$ & Cinchonidine (20 \%) & $-\%$ \\
\hline $\mathrm{H}_{2} \mathrm{O}$ & DBU $(20 \%)$ & $-\%$ \\
\hline $\mathrm{H}_{2} \mathrm{O}$ & Morpholine (20 \%) & $-\%$ \\
\hline $\mathrm{H}_{2} \mathrm{O}$ & Piperidine (20 \%) & $18 \%$ \\
\hline $\mathrm{H}_{2} \mathrm{O}$ & N-Methylpiperidine (20 \%) & $62 \%$ \\
\hline $\mathrm{H}_{2} \mathrm{O}$ & N-Methylpiperidine (40 \%) & $46 \%$ \\
\hline $\mathrm{H}_{2} \mathrm{O}$ & N-Methylpiperidine (10 \%) & $55 \%$ \\
\hline $\mathrm{MeOH}$ & N-Methylpiperidine (20 \%) & $-\%$ \\
\hline
\end{tabular}

${ }^{a}$ yield determined after acetylation.

\section{Characterization of products}


(R)-2-hydroxy-2-((2R,3S,4S,6S)-3,4,6-trihydroxy-6-methyltetrahydro-2H-pyran-2-yl)ethyl acetate (anti-3)<smiles>CC(=O)OCC(O)[C@H]1O[C@@](C)(O)C[C@H](O)[C@@H]1O</smiles>

For further characterization, see "proof of configuration for anti-3" HRMS calcd for $\mathrm{C}_{10} \mathrm{H}_{18} \mathrm{O}_{07} \mathrm{Na}[\mathrm{M}+\mathrm{Na}]^{+}: 273.0945$ found: 273.0946

(R)-2-hydroxy-2-((2R,3S,4R,6S)-3,4,6-trihydroxy-6-methyltetrahydro-2H-pyran-2-yl)ethyl acetate (syn-3)<smiles>CC(=O)OCC(O)[C@H]1O[C@@](C)(O)CC(O)[C@H]1O</smiles>

${ }^{1} \mathrm{H}$ NMR $\left(500 \mathrm{MHz}, \mathrm{D}_{2} \mathrm{O}\right) \delta 4.29(\mathrm{dd}, J=11.7,3.3 \mathrm{~Hz}, 1 \mathrm{H}), 4.24(\mathrm{dd}, J=11.7,7.8 \mathrm{~Hz}, 1 \mathrm{H})$, $4.17(\mathrm{dt}, J=7.8,3.2 \mathrm{~Hz}, 1 \mathrm{H}), 3.89-3.84(\mathrm{~m}, 1 \mathrm{H}), 3.83(\mathrm{dd}, J=10.1,3.0 \mathrm{~Hz}, 1 \mathrm{H}), 3.40$ (dd, $J$ $=9.9,9.2 \mathrm{~Hz}, 1 \mathrm{H}), 2.12(\mathrm{dd}, J=12.4,5.0 \mathrm{~Hz}, 1 \mathrm{H}), 2.12(\mathrm{~s}, 3 \mathrm{H}), 1.58(\mathrm{t}, J=12.4 \mathrm{~Hz}, 1 \mathrm{H})$, $1.43(\mathrm{~s}, 3 \mathrm{H})$.

${ }^{13} \mathrm{C}$ NMR $\left(126 \mathrm{MHz}, \mathrm{D}_{2} \mathrm{O}\right) \delta 174.3,96.8,73.0,71.8,69.5,69.2,65.4,41.6,27.9,20.4$.

HRMS calcd for $\mathrm{C}_{10} \mathrm{H}_{18} \mathrm{O}_{07} \mathrm{Na}[\mathrm{M}+\mathrm{Na}]^{+}: 273.0945$ found: 273.0950

$\underline{(2 R, 3 R, 4 S, 5 S)-7-o x o o c t a n e-1,2,3,4,5-p e n t a y l ~ p e n t a a c e t a t e ~(a n t i-D-r i b o-10)}$<smiles>CC(=O)CC(OC(C)=O)C(C(C)=O)C(OC(C)=O)C(C)=O</smiles>

Yield: $62 \mathrm{mg}, 15 \%$.

${ }^{1} \mathrm{H}$ NMR $\left(500 \mathrm{MHz}, \mathrm{CDCl}_{3}\right) \delta 5.46(\mathrm{dt}, J=8.8,3.7 \mathrm{~Hz}, 1 \mathrm{H}), 5.35(\mathrm{dd}, J=6.3,3.6 \mathrm{~Hz}, 1 \mathrm{H})$, $5.27(\mathrm{~m}, \mathrm{H}), 5.25$ (ddd, $J=6.8,6.4,3.2 \mathrm{~Hz}, 1 \mathrm{H}$ ), 4.34 (dd, $J=12.2,3.2 \mathrm{~Hz}, 1 \mathrm{H}), 4.13$ (dd, $J=12.2,6.7 \mathrm{~Hz}, 1 \mathrm{H}), 2.81$ (dd, $J=17.1,8.8 \mathrm{~Hz}, 1 \mathrm{H}), 2.70(\mathrm{dd}, J=17.1,3.7 \mathrm{~Hz}, 1 \mathrm{H}), 2.15$ (s, 3H), 2.11 (s, 6H), 2.08 (s, 3H), 2.03 (s, 3H), 1.99 (s, 3H).

${ }^{13} \mathrm{C}$ NMR $\left(126 \mathrm{MHz}, \mathrm{CDCl}_{3}\right) \delta 204.0,170.7,170.2,170.0,169.8,169.7,70.8,69.8,69.4$, 68.0, 61.7, 43.0, 30.4, 21.0, 20.9, 20.8, 20.8.

$[\alpha]_{\mathrm{D}}{ }^{25}=+7^{\circ}(\mathrm{c}=1.0$, acetone $)$

HRMS calcd for $\mathrm{C}_{18} \mathrm{H}_{26} \mathrm{O}_{11} \mathrm{Na}[\mathrm{M}+\mathrm{Na}]^{+}: 441.1367$ found: 441.1364 


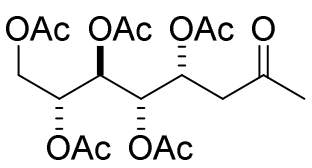

(inseparable mixture with the C-glycoside 75/25)

Yield: $240 \mathrm{mg}, 47$ \% syn-D-ribo-10.

${ }^{1} \mathrm{H}$ NMR $\left(500 \mathrm{MHz}, \mathrm{CDCl}_{3}\right) \delta 5.48(\mathrm{td}, J=7.0,1.9 \mathrm{~Hz}, 1 \mathrm{H}), 5.27(\mathrm{~m}, 2 \mathrm{H}), 5.25-5.19(\mathrm{~m}, 1 \mathrm{H})$, 4.29 (dd, $J=12.0,3.8 \mathrm{~Hz}, 1 \mathrm{H}$ ), 4.12 (dd, $J=12.0,7.4 \mathrm{~Hz}, 1 \mathrm{H}$ ), 2.63 (dd, $J=6.9,2.7 \mathrm{~Hz}, 2 \mathrm{H}$ ), $2.18(\mathrm{~s}, 3 \mathrm{H}), 2.11(\mathrm{~s}, 3 \mathrm{H}), 2.07(\mathrm{~s}, 3 \mathrm{H}), 2.06(\mathrm{~s}, 3 \mathrm{H}), 2.03(\mathrm{~s}, 3 \mathrm{H}), 2.01(\mathrm{~s}, 3 \mathrm{H})$.

${ }^{13} \mathrm{C}$ NMR $\left(126 \mathrm{MHz}, \mathrm{CDCl}_{3}\right) \delta 204.2,170.6,170.3,170.1,170.0,169.7,70.5,69.9,68.6$, $66.8,61.7,43.8,30.2,20.9,20.8,20.8,20.7,20.6$.

$[\alpha]_{\mathrm{D}}^{25}=+39^{\circ}(\mathrm{c}=1.0$, acetone $)$

HRMS calcd for $\mathrm{C}_{18} \mathrm{H}_{26} \mathrm{O}_{11} \mathrm{Na}[\mathrm{M}+\mathrm{Na}]^{+}: 441.1367$ found: 441.1366

$\underline{(2 R, 3 R, 4 S, 5 S)-2-(a c e t o x y m e t h y l)-5-(2-o x o p r o p y l) t e t r a h y d r o f u r a n-3,4-d i y l ~ d i a c e t a t e}$<smiles>CC(=O)CC1OC(COC(C)=O)C(OC(C)=O)C1OC(C)=O</smiles>

${ }^{1} \mathrm{H}$ NMR $\left(500 \mathrm{MHz}, \mathrm{CDCl}_{3}\right) \delta 5.47-5.43(\mathrm{~m}, 1 \mathrm{H}), 5.25(\mathrm{dd}, J=7.4,4.8 \mathrm{~Hz}, 1 \mathrm{H}), 4.61$ (td, $J=$ 6.7, 3.8 Hz, 1H), 4.24 (dd, J=12.0, 3.1 Hz, 1H), 4.13 (ddd, J = 7.6, 4.8, 3.1 Hz, 1H), 4.04 (dd, $J=12.0,4.8 \mathrm{~Hz}, 1 \mathrm{H}), 2.79(\mathrm{dd}, J=17.2,7.0 \mathrm{~Hz}, 1 \mathrm{H}), 2.66(\mathrm{dd}, J=17.2,6.4 \mathrm{~Hz}, 1 \mathrm{H}$ ), $2.13(\mathrm{~s}, 3 \mathrm{H}), 2.08(\mathrm{~s}, 3 \mathrm{H}), 2.04(\mathrm{~s}, 3 \mathrm{H}), 1.99(\mathrm{~s}, 3 \mathrm{H})$.

${ }^{13} \mathrm{C}$ NMR $\left(126 \mathrm{MHz}, \mathrm{CDCl}_{3}\right) \delta 205.2,170.7,169.7,169.6,77.2,75.2,72.4,72.3,63.7,43.5$, $30.5,20.8,20.8,20.7$.

HRMS calcd for $\mathrm{C}_{14} \mathrm{H}_{20} \mathrm{O}_{8} \mathrm{Na}[\mathrm{M}+\mathrm{Na}]^{+}: 339.1050$ found: 339.1051

\section{$\underline{(2 S, 3 S, 4 R, 5 R)-7-o x o o c t a n e-1,2,3,4,5-p e n t a y l ~ p e n t a a c e t a t e ~(a n t i-e n t-10)}$}<smiles>CC(=O)CC(COC(C)=O)[C@H](OC(C)=O)[C@@H](OC(C)=O)C(C)=O</smiles>

Yield: $67 \mathrm{mg}, 16 \%$.

${ }^{1} \mathrm{H}$ NMR $\left(500 \mathrm{MHz}, \mathrm{CDCl}_{3}\right) \delta 5.46(\mathrm{dt}, J=8.8,3.7 \mathrm{~Hz}, 1 \mathrm{H}), 5.35(\mathrm{dd}, J=6.3,3.6 \mathrm{~Hz}, 1 \mathrm{H})$, $5.27(\mathrm{~m}, \mathrm{H}), 5.25$ (ddd, $J=6.8,6.4,3.2 \mathrm{~Hz}, 1 \mathrm{H}$ ), 4.34 (dd, $J=12.2,3.2 \mathrm{~Hz}, 1 \mathrm{H}$ ), 4.13 (dd, $J=12.2,6.7 \mathrm{~Hz}, 1 \mathrm{H}$ ), 2.81 (dd, $J=17.1,8.8 \mathrm{~Hz}, 1 \mathrm{H}$ ), $2.70(\mathrm{dd}, J=17.1,3.7 \mathrm{~Hz}, 1 \mathrm{H}$ ), 2.15 (s, 3H), 2.11 (s, 6H), 2.08 (s, 3H), 2.03 (s, 3H), 1.99 (s, 3H).

${ }^{13} \mathrm{C}$ NMR $\left(126 \mathrm{MHz}, \mathrm{CDCl}_{3}\right) \delta 203.9,170.7,170.2,170.0,169.7,169.7,70.8,69.8,69.4$, 68.0, 61.7, 43.0, 30.3, 21.0, 20.9, 20.8, 20.8. 
$[\alpha]_{D}^{25}=-6^{\circ}(c=1.0$, acetone $)$

HRMS calcd for $\mathrm{C}_{18} \mathrm{H}_{26} \mathrm{O}_{11} \mathrm{Na}[\mathrm{M}+\mathrm{Na}]^{+}: 441.1367$ found: 441.1363

\section{$\underline{(2 S, 3 S, 4 R, 5 S)-7-o x o o c t a n e-1,2,3,4,5-p e n t a y l ~ p e n t a a c e t a t e ~(s y n-e n t-10) ~}$}

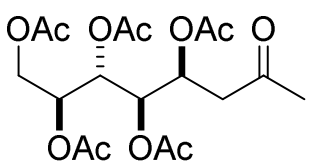

Yield: $177 \mathrm{mg}, 43 \%$.

${ }^{1} \mathrm{H}$ NMR $\left(500 \mathrm{MHz}, \mathrm{CDCl}_{3}\right) \delta 5.48(\mathrm{td}, J=7.0,1.9 \mathrm{~Hz}, 1 \mathrm{H}), 5.27(\mathrm{~d}, J=2.1 \mathrm{~Hz}, 1 \mathrm{H}), 5.25-$ $5.19(\mathrm{~m}, 2 \mathrm{H}), 4.29(\mathrm{dd}, J=12.0,3.8 \mathrm{~Hz}, 1 \mathrm{H}), 4.12(\mathrm{dd}, J=12.0,7.4 \mathrm{~Hz}, 1 \mathrm{H}), 2.63(\mathrm{dd}, J=$ 6.9, $2.7 \mathrm{~Hz}, 2 \mathrm{H}), 2.18(\mathrm{~s}, 3 \mathrm{H}), 2.11(\mathrm{~s}, 3 \mathrm{H}), 2.07$ (s, 3H), 2.06 (s, 3H), 2.03 (s, 3H), 2.01 (s, $3 \mathrm{H})$.

${ }^{13} \mathrm{C}$ NMR $\left(126 \mathrm{MHz}, \mathrm{CDCl}_{3}\right) \delta 204.2,170.7,170.4,170.1,170.0,169.7,70.5,70.0,68.7$, 66.8, 61.7, 43.9, 30.3, 20.9, 20.9, 20.8, 20.8, 20.6.

$[\alpha]_{D}^{25}=-38^{\circ}(c=1.0$, acetone $)$.

HRMS calcd for $\mathrm{C}_{18} \mathrm{H}_{26} \mathrm{O}_{11} \mathrm{Na}[\mathrm{M}+\mathrm{Na}]^{+}: 441.1367$ found: 441.1369

(R)-2-hydroxy-2-((2R,3R,4R,6S)-3,4,6-trihydroxy-6-methyltetrahydro-2H-pyran-2-yl)ethyl acetate<smiles>CC(=O)OCC(O)C1O[C@](C)(O)CC(O)C1O</smiles>

${ }^{1} \mathrm{H}$ NMR $\left(500 \mathrm{MHz}, \mathrm{D}_{2} \mathrm{O}\right) \delta 4.35(\mathrm{dd}, J=11.7,2.5 \mathrm{~Hz}, 1 \mathrm{H}), 4.14(\mathrm{dd}, J=11.7,5.9 \mathrm{~Hz}, 1 \mathrm{H})$, $4.04-3.97(\mathrm{~m}, 3 \mathrm{H}), 3.82(\mathrm{~d}, J=9.4 \mathrm{~Hz}, 1 \mathrm{H}), 2.12(\mathrm{~s}, 3 \mathrm{H}), 1.87(\mathrm{dd}, J=11.1,4.0 \mathrm{~Hz}, 1 \mathrm{H})$, $1.76(\mathrm{t}, J=12.4 \mathrm{~Hz}, 1 \mathrm{H}), 1.42(\mathrm{~s}, 3 \mathrm{H})$.

${ }^{13} \mathrm{C}$ NMR $\left(126 \mathrm{MHz}, \mathrm{D}_{2} \mathrm{O}\right) \delta 174.4,96.9,70.4,66.7,66.3,66.1,65.7,36.6,28.1,20.3$. HRMS calcd for $\mathrm{C}_{10} \mathrm{H}_{18} \mathrm{O}_{07} \mathrm{Na}[\mathrm{M}+\mathrm{Na}]^{+}: 273.0945$ found: 273.0944 


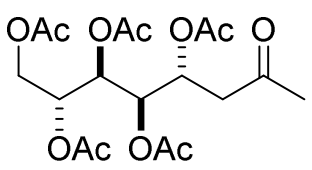

Yield: $176 \mathrm{mg}, 43 \%$

${ }^{1} \mathrm{H}$ NMR $\left(500 \mathrm{MHz}, \mathrm{CDCl}_{3}\right) \delta 5.40$ (dd, $\left.J=8.3,2.9 \mathrm{~Hz}, 1 \mathrm{H}\right), 5.37$ (dd, $\left.J=7.4,4.5 \mathrm{~Hz}, 1 \mathrm{H}\right)$, $5.32(\mathrm{dd}, J=6.8,3.1 \mathrm{~Hz}, 1 \mathrm{H}$ ), 5.08 (ddd, $J=8.3,5.4,2.9 \mathrm{~Hz}, 1 \mathrm{H}$ ), 4.22 (dd, $J=12.7,2.7 \mathrm{~Hz}$, $1 \mathrm{H}), 4.10(\mathrm{dd}, J=12.5,5.4 \mathrm{~Hz}, 1 \mathrm{H}), 2.75(\mathrm{dd}, J=16.7,7.5 \mathrm{~Hz}, 1 \mathrm{H}), 2.68(\mathrm{dd}, J=16.8,4.4$ $\mathrm{Hz}, 1 \mathrm{H}), 2.16$ (s, 3H), 2.10 (s, 3H), 2.07 (s, 3H), 2.06 (s, 6H), 2.01 (s, 3H).

${ }^{13} \mathrm{C} \mathrm{NMR}\left(126 \mathrm{MHz}, \mathrm{CDCl}_{3}\right) \delta 204.1,170.7,170.3,170.1,169.9,70.5,68.4,67.8,66.9,61.9$, $44.5,30.3,21.0,21.0,20.8,20.8$.

$[\alpha]_{D}{ }^{25}=+33^{\circ}(\mathrm{c}=1.0$, acetone $)$

HRMS calcd for $\mathrm{C}_{18} \mathrm{H}_{26} \mathrm{O}_{11} \mathrm{Na}[\mathrm{M}+\mathrm{Na}]^{+}: 441.1367$ found: 441.1364

\section{(2S, 3S, 4S, 5S)-7-oxooctane-1,2,3,4,5-pentayl pentaacetate (anti-ent-11)}<smiles>CC(=O)OCC(OC(C)=O)C(C(C)=O)C(OC(C)=O)C(C)=O</smiles>

Yield: $186 \mathrm{mg}, 45 \%$.

${ }^{1} \mathrm{H}$ NMR $\left(500 \mathrm{MHz}, \mathrm{CDCl}_{3}\right) \delta 5.40(\mathrm{dd}, J=8.3,2.9 \mathrm{~Hz}, 1 \mathrm{H}), 5.37(\mathrm{dd}, J=7.4,4.5 \mathrm{~Hz}, 1 \mathrm{H})$, 5.32 (dd, $J=6.8,3.1 \mathrm{~Hz}, 1 \mathrm{H}$ ), 5.08 (ddd, $J=8.3,5.4,2.9 \mathrm{~Hz}, 1 \mathrm{H}$ ), 4.22 (dd, $J=12.7,2.7 \mathrm{~Hz}$, $1 \mathrm{H}$ ), 4.10 (dd, $J=12.5,5.4 \mathrm{~Hz}, 1 \mathrm{H}$ ), 2.75 (dd, $J=16.7,7.5 \mathrm{~Hz}, 1 \mathrm{H}$ ), 2.68 (dd, $J=16.8,4.4$ $\mathrm{Hz}, 1 \mathrm{H}), 2.16(\mathrm{~s}, 3 \mathrm{H}), 2.10(\mathrm{~s}, 3 \mathrm{H}), 2.07$ (s, 3H), 2.06 (s, 6H), $2.01(\mathrm{~s}, 3 \mathrm{H})$.

${ }^{13} \mathrm{C}$ NMR $\left(126 \mathrm{MHz}, \mathrm{CDCl}_{3}\right) \delta$ 204.1, 170.7, 170.3, 170.1, 169.9, 70.5, 68.4, 67.8, 66.9, 61.9, $44.5,30.3,21.0,21.0,20.8,20.8$.

$[\alpha]_{D}^{25}=-31^{\circ}(\mathrm{c}=1.0$, acetone $)$

HRMS calcd for $\mathrm{C}_{18} \mathrm{H}_{26} \mathrm{O}_{11} \mathrm{Na}[\mathrm{M}+\mathrm{Na}]^{+}: 441.1367$ found: 441.1367

\section{$\underline{(2 R, 3 S, 4 R, 5 R)-7-o x o o c t a n e-1,2,3,4,5-p e n t a y l ~ p e n t a a c e t a t e}$ (anti-D-lyxo-12)}<smiles>CC(=O)CC(COC(C)=O)C(OC(C)=O)C(OC(C)=O)C(C)=O</smiles>

Yield: $78 \mathrm{mg}, 19 \%$.

${ }^{1} \mathrm{H}$ NMR $\left(500 \mathrm{MHz}, \mathrm{CDCl}_{3}\right) \delta 5.33(\mathrm{dt}, J=6.0,2.8 \mathrm{~Hz}, 1 \mathrm{H}), 5.31(\mathrm{dt}, J=8.1,2.6 \mathrm{~Hz}, 1 \mathrm{H}), 5.30$ (dd, $J=4.5,2.5 \mathrm{~Hz}, 1 \mathrm{H}$ ), 5.18 (dd, $J=9.3,2.5 \mathrm{~Hz}, 1 \mathrm{H}$ ), 4.22 (dd, $J=11.5,5.5 \mathrm{~Hz}, 1 \mathrm{H}$ ), 3.88 (dd, $J=11.5,7.2 \mathrm{~Hz}, 1 \mathrm{H}$ ), 2.76 (d, J = 8.0 Hz, 1H), 2.75 (d, J = 3.8 Hz, 1H), $2.16(\mathrm{~s}, 3 \mathrm{H}$ ), $2.14(\mathrm{~s}, 3 \mathrm{H}), 2.04(\mathrm{~s}, 6 \mathrm{H}), 2.01(\mathrm{~s}, 3 \mathrm{H}), 1.94(\mathrm{~s}, 3 \mathrm{H})$. 
${ }^{13} \mathrm{C}$ NMR $\left(126 \mathrm{MHz}, \mathrm{CDCl}_{3}\right) \delta 204.0,170.5,170.4,170.3,170.0,169.6,69.2,68.6,68.0$,

$67.7,61.8,42.0,30.2,20.8,20.8,20.8,20.7,20.7$.

$[\alpha]_{D}^{25}=+37^{\circ}(\mathrm{c}=1.0$, acetone $)$

HRMS calcd for $\mathrm{C}_{18} \mathrm{H}_{26} \mathrm{O}_{11} \mathrm{Na}[\mathrm{M}+\mathrm{Na}]^{+}: 441.1367$ found: 441.1365

$\underline{(2 R, 3 S, 4 R, 5 S)-7-o x o o c t a n e-1,2,3,4,5-p e n t a y l ~ p e n t a a c e t a t e}($ syn-D-lyxo-12)<smiles>CC(=O)CC(COC(C)=O)C(OC(C)=O)[C@@H](OC(C)=O)C(C)=O</smiles>

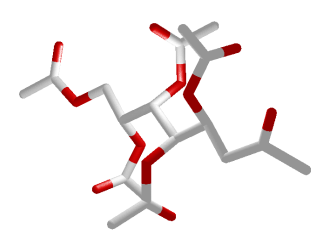

Yield: $184 \mathrm{mg}, 44 \%$.

${ }^{1} \mathrm{H}$ NMR $\left(500 \mathrm{MHz}, \mathrm{CDCl}_{3}\right) \delta 5.37(\mathrm{t}, J=6.9 \mathrm{~Hz}, 1 \mathrm{H}), 5.33-5.26(\mathrm{~m}, 2 \mathrm{H}), 5.19(\mathrm{~d}, J=10.0$ $\mathrm{Hz}, 1 \mathrm{H}), 4.26$ (dd, $J=11.7,4.4 \mathrm{~Hz}, 1 \mathrm{H}), 3.80(\mathrm{dd}, J=11.6,8.0 \mathrm{~Hz}, 1 \mathrm{H}), 2.55(\mathrm{~d}, J=6.9 \mathrm{~Hz}$, 2H), 2.10 (s, 3H), 2.08 (s, 3H), 2.07 (s, 3H), 2.02 (s, 3H), 2.01 (s, 3H), 1.98 (s, 3H).

${ }^{13} \mathrm{C}$ NMR $\left(126 \mathrm{MHz}, \mathrm{CDCl}_{3}\right) \delta 204.2,170.6,170.5,170.4,170.2,170.0,69.1,67.9,67.7$, $66.5,62.4,43.8,30.2,20.9,20.8,20.7,20.7$.

$[\alpha]_{D}^{25}=+14^{\circ}(\mathrm{c}=1.0$, acetone $)$

HRMS calcd for $\mathrm{C}_{18} \mathrm{H}_{26} \mathrm{O}_{11} \mathrm{Na}[\mathrm{M}+\mathrm{Na}]^{+}: 441.1367$ found: 441.1364

(R)-2-hydroxy-2-((2S,3S,4S,6R)-3,4,6-trihydroxy-6-methyltetrahydro-2H-pyran-2-yl)ethyl acetate<smiles>CC(=O)OCC(O)C1(O)O[C@](C)(O)C[C@@H](O)[C@@H]1O</smiles>

${ }^{1} \mathrm{H}$ NMR $\left(500 \mathrm{MHz}, \mathrm{D}_{2} \mathrm{O}\right) \delta 4.31(\mathrm{dd}, J=11.9,3.0 \mathrm{~Hz}, 1 \mathrm{H}), 4.19(\mathrm{dd}, J=11.9,5.7 \mathrm{~Hz}, 1 \mathrm{H})$, 4.09 (ddd, $J=8.6,5.7,2.9 \mathrm{~Hz}, 1 \mathrm{H}$ ), 3.99 (ddd, $J=11.9,5.2,2.8 \mathrm{~Hz}, 1 \mathrm{H}$ ), 3.93 (dd, $J=7.7$, $1.0 \mathrm{~Hz}, 1 \mathrm{H}$ ), 3.85 (d, $J=2.9 \mathrm{~Hz}, 1 \mathrm{H}), 2.12(\mathrm{~s}, 3 \mathrm{H}), 1.87$ (dd, $J=13.1,5.0 \mathrm{~Hz}, 1 \mathrm{H}), 1.77$ (dd, $J$ $=13.1,11.9 \mathrm{~Hz}, 1 \mathrm{H}), 1.47(\mathrm{~s}, 3 \mathrm{H})$.

${ }^{13} \mathrm{C}$ NMR $\left(126 \mathrm{MHz}, \mathrm{D}_{2} \mathrm{O}\right) \delta 174.2,96.8,71.5,69.2,67.1,66.0,65.0,36.5,28.2,20.2$. HRMS calcd for $\mathrm{C}_{10} \mathrm{H}_{18} \mathrm{O}_{07} \mathrm{Na}[\mathrm{M}+\mathrm{Na}]^{+}: 273.0945$ found: 273.0949 


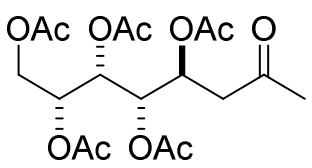

Yield: $146 \mathrm{mg}, 35 \%$.

${ }^{1} \mathrm{H}$ NMR $\left(500 \mathrm{MHz}, \mathrm{CDCl}_{3}\right) \delta 5.36(\mathrm{dd}, J=9.8,4.9 \mathrm{~Hz}, 1 \mathrm{H}), 5.33(\mathrm{dt}, J=8.6,4.6 \mathrm{~Hz}, 1 \mathrm{H})$, $5.29(\mathrm{t}, J=5.4 \mathrm{~Hz}, 1 \mathrm{H}), 5.24(\mathrm{td}, J=5.8,4.3 \mathrm{~Hz}, 1 \mathrm{H}), 4.32(\mathrm{dd}, J=12.0,4.3 \mathrm{~Hz}, 1 \mathrm{H}), 3.99$ (dd, $J=12.0,6.1 \mathrm{~Hz}, 1 \mathrm{H}), 2.80$ (dd, $J=17.0,7.9 \mathrm{~Hz}, 1 \mathrm{H}$ ), 2.66 (dd, $J=17.0,4.2 \mathrm{~Hz}, 1 \mathrm{H}$ ), $2.16(\mathrm{~s}, 3 \mathrm{H}), 2.10(\mathrm{~s}, 3 \mathrm{H}), 2.09(\mathrm{~s}, 3 \mathrm{H}), 2.07$ (s, 3H), $2.04(\mathrm{~s}, 3 \mathrm{H}), 1.97(\mathrm{~s}, 3 \mathrm{H})$.

${ }^{13} \mathrm{C}$ NMR $\left(126 \mathrm{MHz}, \mathrm{CDCl}_{3}\right) \delta 204.0,170.5,170.2,170.0,169.9,169.8,71.0,69.1,68.6$, $67.4,61.8,43.5,30.3,20.8,20.8,20.8,20.7$.

$[\alpha]_{D}^{25}=-3^{\circ}(c=1.0$, acetone $)$

HRMS calcd for $\mathrm{C}_{18} \mathrm{H}_{26} \mathrm{O}_{11} \mathrm{Na}[\mathrm{M}+\mathrm{Na}]^{+}: 441.1367$ found: 441.1369

(2R,3S, 5S)-7-oxooctane-1,2,3,5-tetrayl tetraacetate (D-deoxyribo-14)<smiles>CC(=O)CC(CC(C)=O)C(COC(C)=O)OC(C)=O</smiles>

Yield: $269 \mathrm{mg}, 75 \%$.

${ }^{1} \mathrm{H}$ NMR $\left(500 \mathrm{MHz}, \mathrm{CDCl}_{3}\right) \delta 5.14-5.09(\mathrm{~m}, 1 \mathrm{H}), 5.09-5.04(\mathrm{~m}, 2 \mathrm{H}), 4.17(\mathrm{dd}, J=12.1,3.1$ $\mathrm{Hz}, 1 \mathrm{H}$ ), 4.03 (dd, $J=12.1,6.9 \mathrm{~Hz}, 1 \mathrm{H}), 2.71(\mathrm{dd}, J=16.6,5.8 \mathrm{~Hz}, 1 \mathrm{H}), 2.57$ (dd, $J=16.6$, $6.8 \mathrm{~Hz}, 1 \mathrm{H}), 2.07$ (s, 3H), $1.99(\mathrm{~s}, 3 \mathrm{H}), 1.96(\mathrm{~s}, 3 \mathrm{H}), 1.96(\mathrm{~s}, 3 \mathrm{H}), 1.92(\mathrm{~s}, 3 \mathrm{H}), 1.88$ (ddd, J = 12.9, 10.3, 3.0 Hz, 1H), 1.75 (ddd, $J=12.9,10.1,2.3 \mathrm{~Hz}, 1 \mathrm{H}$ ).

${ }^{13} \mathrm{C}$ NMR $\left(126 \mathrm{MHz}, \mathrm{CDCl}_{3}\right) \delta 205.0,170.5,170.2,170.1,170.1,71.8,67.8,65.9,61.8,47.7$, 34.0, 30.4, 20.8, 20.8, 20.7, 20.6.

$[\alpha]_{D}^{25}=-1^{\circ}(c=1.0$, acetone $)$

HRMS calcd for $\mathrm{C}_{16} \mathrm{H}_{24} \mathrm{O}_{9} \mathrm{Na}[\mathrm{M}+\mathrm{Na}]^{+}: 383.1313$ found: 383.1311 
$\underline{(2 R, 3 R)-2,3-d i h y d r o x y-3-((2 R, 3 S, 4 S, 6 R)-3,4,6 \text {-trihydroxy-6-methyltetrahydro-2H-pyran-2- }}$

yl)propyl acetate

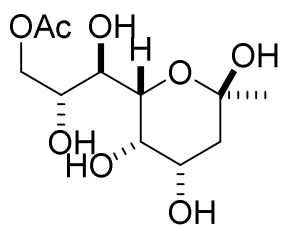

${ }^{1} \mathrm{H}$ NMR $\left(500 \mathrm{MHz}, \mathrm{D}_{2} \mathrm{O}\right) \delta 4.39-4.30(\mathrm{~m}, 1 \mathrm{H}), 4.23-4.16(\mathrm{~m}, 1 \mathrm{H}), 4.03-3.98(\mathrm{~m}, 1 \mathrm{H})$, $3.96-3.93(\mathrm{~m}, 2 \mathrm{H}), 3.78-3.75(\mathrm{~m}, 1 \mathrm{H}), 3.64(\mathrm{dd}, J=6.6,3.0 \mathrm{~Hz}, 1 \mathrm{H}), 2.13(\mathrm{~s}, 3 \mathrm{H}), 1.87$ (dd, $J=13.1,5.2 \mathrm{~Hz}, 1 \mathrm{H}), 1.83-1.77$ (dd, $J=13.1,12.0,1 \mathrm{H}), 1.47(\mathrm{~s}, 3 \mathrm{H})$.

${ }^{13} \mathrm{C}$ NMR $\left(126 \mathrm{MHz}, \mathrm{D}_{2} \mathrm{O}\right) \delta 174.4,96.8,74.0,70.3,69.6,68.4,66.9,66.0,46.9,30.0,20.3$. HRMS calcd for $\mathrm{C}_{10} \mathrm{H}_{18} \mathrm{O}_{07} \mathrm{Na}[\mathrm{M}+\mathrm{Na}]^{+}: 273.0945$ found: 273.0948

$\underline{(2 R, 3 R, 4 R, 5 S, 6 R)-8-o x o n o n a n e-1,2,3,4,5,6 \text {-hexayl hexaacetate (syn-D-gluco-20) }}$<smiles></smiles>

Yield: $20 \mathrm{mg}, 5 \%$.

${ }^{1} \mathrm{H}$ NMR $\left(500 \mathrm{MHz}, \mathrm{CDCl}_{3}\right) \delta 5.53$ (ddd, $\left.J=7.1,6.4,2.2 \mathrm{~Hz}, 1 \mathrm{H}\right), 5.47$ (dd, $J=8.4,2.4 \mathrm{~Hz}$, 1H), 5.36 (dd, $J=8.8,2.4 \mathrm{~Hz}, 1 \mathrm{H}$ ), 5.19 (dd, $J=8.8,2.2 \mathrm{~Hz}, 1 \mathrm{H}$ ), 5.02 (ddd, $J=8.1,5.1,2.8$ $\mathrm{Hz}, 1 \mathrm{H}$ ), 4.22 (dd, $J=12.5,2.8 \mathrm{~Hz}, 1 \mathrm{H}), 4.10$ (dd, $J=12.5,5.1 \mathrm{~Hz}, 1 \mathrm{H}), 2.68$ (dd, $J=17.4$, $6.3 \mathrm{~Hz}, 1 \mathrm{H}$ ), 2.60 (dd, J = 17.4, 7.1 Hz, 1H), 2.22 (s, 3H), 2.10 (s, 3H), 2.09 (s, 3H), 2.07 (s, $3 \mathrm{H}), 2.06(\mathrm{~s}, 3 \mathrm{H}), 2.04(\mathrm{~s}, 3 \mathrm{H}), 2.03(\mathrm{~s}, 3 \mathrm{H})$.

${ }^{13} \mathrm{C}$ NMR $\left(126 \mathrm{MHz}, \mathrm{CDCl}_{3}\right)$ ठ 204.1, 170.8, 170.3, 170.3, 170.0, 169.9, 71.2, 68.6, 68.6, 67.9, 67.3, 61.7, 44.1, 30.1, 20.9, 20.9, 20.8, 20.7, 20.6.

$[\alpha]_{D}^{25}=-2^{\circ}(c=1.0$, acetone $)$

HRMS calcd for $\mathrm{C}_{21} \mathrm{H}_{30} \mathrm{O}_{13} \mathrm{Na}[\mathrm{M}+\mathrm{Na}]^{+}: 513.1579$ found: 513.1578

$(2 R, 3 R, 4 R, 5 S, 6 S)$-8-oxononane-1, 2,3,4,5,6-hexayl hexaacetate (anti-D-gluco-20)<smiles>CC(=O)CC(OC(C)=O)[C@H](OC(C)=O)[C@@H](OC(C)=O)[C@H](OC(C)=O)C(C)=O</smiles>

Yield: $83 \mathrm{mg}, 17 \%$.

${ }^{1} \mathrm{H}$ NMR $\left(500 \mathrm{MHz}, \mathrm{CDCl}_{3}\right) \delta 5.43(\mathrm{dd}, J=6.6,4.7 \mathrm{~Hz}, 1 \mathrm{H}), 5.40(\mathrm{dd}, J=6.3,4.6 \mathrm{~Hz}, 1 \mathrm{H})$, 5.30 (dt, $J=2.9,1.2 \mathrm{~Hz}, 1 \mathrm{H}), 5.29(\mathrm{t}, J=4.0 \mathrm{~Hz}, 1 \mathrm{H}), 5.03$ (ddd, $J=6.5,5.8,3.8 \mathrm{~Hz}, 1 \mathrm{H}$ ), 4.26 (dd, $J=12.3,3.8 \mathrm{~Hz}, 1 \mathrm{H}$ ), 4.09 (dd, $J=12.3,5.7 \mathrm{~Hz}, 1 \mathrm{H}$ ), 2.80 (dd, $J=17.1,7.9 \mathrm{~Hz}$, 1H), 2.71 (dd, J = 17.1, 4.4 Hz, 1H), 2.18 (s, 3H), 2.15 (s, 3H), 2.10 (s, 3H), 2.09 (s, 3H), 2.04 $(\mathrm{s}, 3 \mathrm{H}), 2.03(\mathrm{~s}, 3 \mathrm{H}), 1.96(\mathrm{~s}, 3 \mathrm{H})$. 
${ }^{13} \mathrm{C}$ NMR $\left(126 \mathrm{MHz}, \mathrm{CDCl}_{3}\right) \delta 204.0,170.7,170.2,170.1,170.1,170.0,169.8,71.0,68.9$, $68.8,68.4,67.5,61.6,43.3,30.4,20.9,20.8,20.8,20.8,20.7$.

$[\alpha]_{D}^{25}=+46^{\circ}(\mathrm{c}=1.0$, acetone $)$

HRMS calcd for $\mathrm{C}_{21} \mathrm{H}_{30} \mathrm{O}_{13} \mathrm{Na}[\mathrm{M}+\mathrm{Na}]^{+}: 513.1579$ found: 513.1576

(2R,3S, 4R, 5S, 6S)-8-oxononane-1,2,3,4,5,6-hexayl hexaacetate (anti-D-galacto-21)<smiles>CC(=O)CC(OC(C)=O)[C@H](OC(C)=O)C(OC(C)=O)C(OC(C)=O)C(C)=O</smiles>

Yield: $199 \mathrm{mg}, 41 \%$.

${ }^{1} \mathrm{H}$ NMR $\left(500 \mathrm{MHz}, \mathrm{CDCl}_{3}\right) \delta 5.43(\mathrm{dd}, J=9.9,2.1 \mathrm{~Hz}, 1 \mathrm{H}), 5.29(\mathrm{dd}, J=7.0,2.1 \mathrm{~Hz}, 1 \mathrm{H})$, $5.26(\mathrm{dd}, J=9.9,2.0 \mathrm{~Hz}, 1 \mathrm{H}), 5.21-5.18(\mathrm{~m}, 1 \mathrm{H}), 5.19(\mathrm{dd}, J=6.7,2.1 \mathrm{~Hz}, 1 \mathrm{H}), 4.25(\mathrm{dd}, J$ $=11.7,5.0 \mathrm{~Hz}, 1 \mathrm{H}$ ), 3.81 (dd, $J=11.7,7.3 \mathrm{~Hz}, 1 \mathrm{H}$ ), 2.74 (dd, J = 16.8, 7.2 Hz, 1H), 2.65 (dd, $J=16.8,4.8 \mathrm{~Hz}, 1 \mathrm{H}), 2.13(\mathrm{~s}, 3 \mathrm{H}), 2.10(\mathrm{~s}, 3 \mathrm{H}), 2.08(\mathrm{~s}, 3 \mathrm{H}), 2.06(\mathrm{~s}, 3 \mathrm{H}), 2.06(\mathrm{~s}, 3 \mathrm{H}), 2.00$ $(\mathrm{s}, 3 \mathrm{H}), 1.99(\mathrm{~s}, 3 \mathrm{H})$.

${ }^{13} \mathrm{C}$ NMR $\left(126 \mathrm{MHz}, \mathrm{CDCl}_{3}\right) \delta 204.0,170.6,170.5,170.4,170.0,169.9,169.8,70.0,67.8$, $67.7,67.2,66.7,62.3,44.5,30.3,21.0,20.9,20.9,20.8,20.8,20.8$.

$[\alpha]_{\mathrm{D}}^{25}=+4^{\circ}(\mathrm{c}=1.0$, acetone $)$

HRMS calcd for $\mathrm{C}_{21} \mathrm{H}_{30} \mathrm{O}_{13} \mathrm{Na}[\mathrm{M}+\mathrm{Na}]^{+}: 513.1579$ found: 513.1576

\section{$\underline{(2 R, 3 R, 4 R, 5 R, 6 R)-8-o x o n o n a n e-1,2,3,4,5,6 \text {-hexayl hexaacetate (anti-D-manno-22) }}$}<smiles></smiles>

Yield: $111 \mathrm{mg}, 23 \%$

${ }^{1} \mathrm{H}$ NMR $\left(500 \mathrm{MHz}, \mathrm{CDCl}_{3}\right) \delta 5.36(\mathrm{dd}, J=9.2,2.0 \mathrm{~Hz}, 1 \mathrm{H}), 5.24(\mathrm{dt}, J=4.0,2.4 \mathrm{~Hz}, 1 \mathrm{H})$, 5.24 (dd, $J=9.8,2.0 \mathrm{~Hz}, 1 \mathrm{H}$ ), 5.20 (dd, $J=9.9,2.0 \mathrm{~Hz}, 1 \mathrm{H}$ ), 5.04 (ddd, $J=9.1,5.2,2.8 \mathrm{~Hz}$, 1H), 4.16 (dd, $J=12.6,2.8 \mathrm{~Hz}, 1 \mathrm{H}$ ), 3.99 (dd, $J=12.6,5.2 \mathrm{~Hz}, 1 \mathrm{H}$ ), 2.80 (dd, $J=16.9,2.9$ Hz, 1H), 2.74 (dd, J = 17.0, $9.5 \mathrm{~Hz}, 1 \mathrm{H}), 2.12$ (s, 3H), 2.12 (s, 3H), 2.04 (s, 3H), $2.04(\mathrm{~s}, 3 \mathrm{H})$, $2.03(\mathrm{~s}, 3 \mathrm{H}), 2.02(\mathrm{~s}, 3 \mathrm{H}), 1.92(\mathrm{~s}, 3 \mathrm{H})$.

${ }^{13} \mathrm{C}$ NMR $\left(126 \mathrm{MHz}, \mathrm{CDCl}_{3}\right) \delta 204.1,170.6,170.5,170.0,170.0,169.9,169.7,68.8,68.2$, $67.9,67.7,67.2,62.0,41.7,30.1,20.9,20.9,20.8,20.8,20.7$.

$[\alpha]_{\mathrm{D}}^{25}=+25^{\circ}(\mathrm{c}=1.0$, acetone $)$

HRMS calcd for $\mathrm{C}_{21} \mathrm{H}_{30} \mathrm{O}_{13} \mathrm{Na}[\mathrm{M}+\mathrm{Na}]^{+}: 513.1579$ found: 513.1580 
<smiles>CC(=O)CC(COC(C)=O)C(OC(C)=O)C(OC(C)=O)C(OC(C)=O)C(C)=O</smiles>

Inseparable mixture with hemiketal 60/40.

Yield: $251 \mathrm{mg}, 53 \%$.

${ }^{1} \mathrm{H}$ NMR $\left(500 \mathrm{MHz}, \mathrm{CDCl}_{3}\right) \delta 5.45(\mathrm{dd}, J=10.1,1.9 \mathrm{~Hz}, 1 \mathrm{H}), 5.35(\mathrm{dd}, J=9.0,1.9 \mathrm{~Hz}, 1 \mathrm{H})$, 5.19 (ddd, $J=8.2,5.6,1.4 \mathrm{~Hz}, 1 \mathrm{H}$ ), 5.10 (dd, $J=10.1,1.5 \mathrm{~Hz}, 1 \mathrm{H}$ ), 4.99 (ddd, $J=8.9,5.1$, $2.8 \mathrm{~Hz}, 1 \mathrm{H}), 4.17(\mathrm{dd}, J=12.5,2.7 \mathrm{~Hz}, 1 \mathrm{H}), 4.01(\mathrm{dd}, J=12.6,5.1 \mathrm{~Hz}, 1 \mathrm{H}), 2.58(\mathrm{dd}, J=$ 17.2, $5.5 \mathrm{~Hz}, 1 \mathrm{H}), 2.53(\mathrm{dd}, J=17.1,8.1 \mathrm{~Hz}, 1 \mathrm{H}), 2.10(\mathrm{~s}, 3 \mathrm{H}), 2.06(\mathrm{~s}, 6 \mathrm{H}), 2.03(\mathrm{~s}, 6 \mathrm{H})$, $2.02(\mathrm{~s}, 3 \mathrm{H}), 2.01(\mathrm{~s}, 3 \mathrm{H})$.

${ }^{13} \mathrm{C}$ NMR $\left(126 \mathrm{MHz}, \mathrm{CDCl}_{3}\right) \delta$ 204.3, 170.6, 170.3, 170.1, 170.0, 169.9, 68.9, 68.1, 67.5, 66.9, 66.8, 62.0, 43.6, 30.1, 21.0, 21.0, 20.9, 20.9, 20.8, 20.7.

HRMS calcd for $\mathrm{C}_{21} \mathrm{H}_{30} \mathrm{O}_{13} \mathrm{Na}[\mathrm{M}+\mathrm{Na}]^{+}: 513.1579$ found: 513.1578

$(1 R, 2 R)-1-((2 R, 3 R, 4 S, 6 R)-3,4-d i a c e t o x y-6$-hydroxy-6-methyltetrahydro-2H-pyran-2-

yl)propane-1,2,3-triyl triacetate (syn-D-manno-22 (hemiketal)

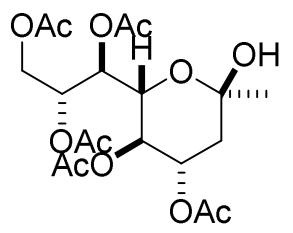

${ }^{1} \mathrm{H}$ NMR $\left(500 \mathrm{MHz}, \mathrm{CDCl}_{3}\right) \delta 5.38$ (ddd, $\left.J=7.6,6.5,2.2 \mathrm{~Hz}, 1 \mathrm{H}\right), 5.26(\mathrm{~m}, 1 \mathrm{H}), 5.25$ (dd, $J=$ 7.6, $1.9 \mathrm{~Hz}, 1 \mathrm{H}$ ), $4.79(\mathrm{t}, J=9.8 \mathrm{~Hz}, 1 \mathrm{H}), 4.37(\mathrm{dd}, J=12.4,2.2 \mathrm{~Hz}, 1 \mathrm{H}), 4.11$ (dd, $J=10.2$, $1.9 \mathrm{~Hz}, 1 \mathrm{H}$ ), 4.07 (dd, $J=12.4,6.5 \mathrm{~Hz}, 1 \mathrm{H}), 2.24$ (dd, $J=12.9,5.4 \mathrm{~Hz}, 1 \mathrm{H}), 2.08(\mathrm{~s}, 3 \mathrm{H})$, 2.07 (s, 3H), 2.02 (s, 3H), 1.99 (s, 3H), $1.96(\mathrm{~s}, 3 \mathrm{H}), 1.67$ (t, J = $12.2 \mathrm{~Hz}, 1 \mathrm{H}) 1.48(\mathrm{~s}, 3 \mathrm{H})$.

${ }^{13} \mathrm{C}$ NMR $\left(126 \mathrm{MHz}, \mathrm{CDCl}_{3}\right) \delta 170.9,170.6,170.3,170.2,170.1,97.0,70.4,69.7,68.7,68.0$, $67.4,62.9,39.8,29.3,21.2,21.1,20.7,20.7$.

HRMS calcd for $\mathrm{C}_{19} \mathrm{H}_{28} \mathrm{O}_{13} \mathrm{Na}[\mathrm{M}+\mathrm{Na}]^{+}: 471.1473$ found: 471.1476

$\underline{(2 R, 3 S, 4 R, 6 R)-8-o x o n o n a n e-1,2,3,4,6 \text {-pentayl pentaacetate (D-deoxygluco-23) }}$<smiles>CC(=O)CC(CC(OC(C)=O)[C@H](OC(C)=O)C(C)=O)OC(C)=O</smiles>

Yield: $360 \mathrm{mg}, 84 \%$.

${ }^{1} \mathrm{H}$ NMR $\left(500 \mathrm{MHz}, \mathrm{CDCl}_{3}\right) \delta 5.34(\mathrm{dt}, J=10.6,2.9 \mathrm{~Hz}, 1 \mathrm{H}), 5.23(\mathrm{dd}, J=8.5,2.8 \mathrm{~Hz}, 1 \mathrm{H})$, 5.04 (ddd, $J=10.8,6.8,2.4 \mathrm{~Hz}, 1 \mathrm{H}$ ), $5.04-4.99$ (m, 1H), 4.21 (dd, $J=12.5,2.7 \mathrm{~Hz}, 1 \mathrm{H}$ ), $4.12(\mathrm{dd}, J=12.5,4.8 \mathrm{~Hz}, 1 \mathrm{H}), 2.78(\mathrm{dd}, J=16.7,5.2 \mathrm{~Hz}, 1 \mathrm{H}), 2.59$ (dd, $J=16.7,7.3 \mathrm{~Hz}$, 
$1 \mathrm{H}), 2.12(\mathrm{~s}, 3 \mathrm{H}), 2.11(\mathrm{~s}, 3 \mathrm{H}), 2.04(\mathrm{~s}, 3 \mathrm{H}), 2.04(\mathrm{~s}, 3 \mathrm{H}), 2.03(\mathrm{~s}, 3 \mathrm{H}), 2.00(\mathrm{~s}, 3 \mathrm{H}), 1.92-$ 1.85 (ddd, $J=14.5,10.6,3.0,1 \mathrm{H}$ ), 1.74 (ddd, $J=14.5,10.3,2.9 \mathrm{~Hz}, 1 \mathrm{H}$ ).

${ }^{13} \mathrm{C}$ NMR $\left(126 \mathrm{MHz}, \mathrm{CDCl}_{3}\right) \delta 205.1,170.7,170.5,170.4,170.1,170.0,71.0,68.5,66.6$, $66.3,61.9,47.9,35.2,30.6,21.1,21.0,20.8,20.8,20.8$.

$[\alpha]_{D}^{25}=+42^{\circ}(c=1.0$, acetone $)$

HRMS calcd for $\mathrm{C}_{19} \mathrm{H}_{28} \mathrm{O}_{11} \mathrm{Na}[\mathrm{M}+\mathrm{Na}]^{+}: 455.1524$ found: 455.1528

\section{$\underline{\text { (2R,3R,4R,6R)-8-oxononane-1,2,3,4,6-pentayl pentaacetate D-deoxygalacto-24 }}$}

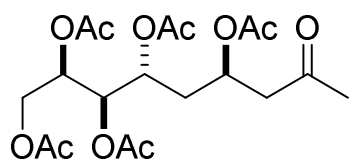

Yield: $383 \mathrm{mg}, 89 \%$.

${ }^{1} \mathrm{H}$ NMR $\left(500 \mathrm{MHz}, \mathrm{CDCl}_{3}\right) \delta 5.27-5.21(\mathrm{~m}, 2 \mathrm{H}), 5.12$ (tdd, $\left.J=6.8,6.3,2.9 \mathrm{~Hz}, 1 \mathrm{H}\right), 5.03$ (ddd, $J=10.4,5.5,2.6 \mathrm{~Hz}, 1 \mathrm{H}$ ), 4.17 (dd, $J=11.8,4.5 \mathrm{~Hz}, 1 \mathrm{H}$ ), 3.98 (dd, $J=11.8,6.1 \mathrm{~Hz}$, 1H), $2.75(\mathrm{dd}, J=16.7,5.7 \mathrm{~Hz}, 1 \mathrm{H}), 2.62(\mathrm{dd}, J=16.6,6.9 \mathrm{~Hz}, 1 \mathrm{H}), 2.11(\mathrm{~s}, 3 \mathrm{H}), 2.09$ (s, $3 \mathrm{H}$ ), $2.04(\mathrm{~s}, 3 \mathrm{H}), 2.02(\mathrm{~s}, 3 \mathrm{H}), 1.99(\mathrm{~s}, 3 \mathrm{H}), 1.98(\mathrm{~s}, 3 \mathrm{H}), 1.92(\mathrm{ddd}, J=14.7,10.5,3.0 \mathrm{~Hz}$, $1 \mathrm{H}$ ), 1.78 (ddd, $J=14.7,10.2,2.6 \mathrm{~Hz}, 1 \mathrm{H}$ ).

${ }^{13} \mathrm{C}$ NMR $\left(126 \mathrm{MHz}, \mathrm{CDCl}_{3}\right) \delta 205.0,170.6,170.4,170.2,170.2,170.0,71.3,68.6,67.0$, $66.0,61.9,47.8,34.2,30.6,21.0,20.8,20.8,20.8,20.7$.

$[\alpha]_{\mathrm{D}}^{25}=+27^{\circ}(\mathrm{c}=1.0$, acetone $)$

HRMS calcd for $\mathrm{C}_{19} \mathrm{H}_{28} \mathrm{O}_{11} \mathrm{Na}[\mathrm{M}+\mathrm{Na}]^{+}: 455.1524$ found: 455.1527

(2R,3S)-3-((2R,4R,5S,6R)-4,6-dihydroxy-5,6-dimethyltetrahydro-2H-pyran-2-yl)-2,3dihydroxypropyl acetate<smiles>CC(=O)OC[C@H](O)[C@H](O)[C@H]1C[C@@H](O)[C@@H](C)[C@](C)(O)O1</smiles>

${ }^{1} \mathrm{H}$ NMR $\left(500 \mathrm{MHz}, \mathrm{D}_{2} \mathrm{O}\right) \delta 4.20-4.14(\mathrm{~m}, 2 \mathrm{H}), 4.04$ (ddd, J = 6.4, 5.4, 3.0 Hz, $1 \mathrm{H}$ ), 3.96 (ddd, $J=11.8,7.7,2.0 \mathrm{~Hz}, 1 \mathrm{H}$ ), 3.67 (td, $J=11.0,4.6 \mathrm{~Hz}, 1 \mathrm{H}$ ), 3.50 (dd, $J=7.7,3.0 \mathrm{~Hz}, 1 \mathrm{H}$ ), 2.17 (ddd, $J=12.3,4.6,2.1 \mathrm{~Hz}, 1 \mathrm{H}), 2.10(\mathrm{~s}, 3 \mathrm{H}), 1.50-1.43(\mathrm{~m}, 1 \mathrm{H}), 1.41$ (s, 3H), 1.40 $1.26(\mathrm{~m}, 1 \mathrm{H}), 1.04(\mathrm{~d}, J=6.8 \mathrm{~Hz}, 3 \mathrm{H})$.

${ }^{13}$ C NMR $\left(126 \mathrm{MHz}, \mathrm{D}_{2} \mathrm{O}\right) \delta 174.2,99.9,72.5,69.2,67.8,67.6,65.9,46.3,36.0,26.4,20.3$, 11.4 .

HRMS calcd for $\mathrm{C}_{12} \mathrm{H}_{22} \mathrm{O}_{7} \mathrm{Na}[\mathrm{M}+\mathrm{Na}]^{+}: 301.1258$ found: 301.1264 
<smiles>CC(=O)OCC(CC(C(C)=O)C(OC(C)=O)C(C)=O)C(C)C(C)=O</smiles>

inseparable mixture of syn / anti 24 (75/25); yield $344 \mathrm{mg}, 58 \%$

${ }^{1} \mathrm{H}$ NMR $\left(500 \mathrm{MHz}, \mathrm{CDCl}_{3}\right) \delta 5.24(\mathrm{~m}, 2 \mathrm{H}), 5.29$ ( ddd, $\left.J=4.6,6.3,8.9,1 \mathrm{H}\right), 5.14$ (ddd, $J=$ $5.5,6.7,9.0,1 \mathrm{H}), 4.98(\mathrm{ddd}, J=4.9,6.3,8.9,1 \mathrm{H}), 4.14(\mathrm{dd}, J=11.8,4.6 \mathrm{~Hz}, 1 \mathrm{H}), 3.97(\mathrm{dd}, J$ $=11.9,6.0 \mathrm{~Hz}, 1 \mathrm{H}), 2.73(\mathrm{dq}, J=7.1,5.5 \mathrm{~Hz}, 1 \mathrm{H}), 2.14(\mathrm{~s}, 3 \mathrm{H}), 2.07(\mathrm{~s}, 3 \mathrm{H}), 2.03(\mathrm{~s}, 3 \mathrm{H})$, $2.01(\mathrm{~s}, 3 \mathrm{H}), 1.98(\mathrm{~s}, 3 \mathrm{H}), 1.97(\mathrm{~s}, 3 \mathrm{H}), 1.85(\mathrm{~m}, 1 \mathrm{H}), 1.79$ (ddd, $J=4.2,6.8,9.2,1 \mathrm{H}), 1.02$ (d, $J=7.1 \mathrm{~Hz}, 3 \mathrm{H})$.

${ }^{13}$ C NMR $\left(126 \mathrm{MHz}, \mathrm{CDCl}_{3}\right) \delta 209.1,170.5,170.4,170.1,170.1,170.0,71.2,69.3,68.6$, 67.2, 61.8, 50.3, 31.7, 29.8, 20.8, 20.8, 20.7, 20.7, 11.8 .

HRMS calcd for $\mathrm{C}_{20} \mathrm{H}_{31} \mathrm{O}_{11}[\mathrm{M}+\mathrm{H}]^{+}: 447.1861$ found: 447.1870

$\underline{(2 R, 3 R, 4 R, 6 R, 7 R)-7-\text { methyl-pentacetoxy-nonan-8-one (anti-25) }}$<smiles>CC(=O)OCC(CC(OC(C)=O)C(OC(C)=O)C(C)=O)[C@@H](C)C(C)=O</smiles>

Yield: $15 \%$

${ }^{1} \mathrm{H}$ NMR $\left(500 \mathrm{MHz}, \mathrm{CDCl}_{3}\right) \delta 5.24-5.18(\mathrm{~m}, 2 \mathrm{H}), 5.08$ (ddd, $\left.J=10.7,5.6,2.7 \mathrm{~Hz}, 1 \mathrm{H}\right), 4.99$ $-4.93(\mathrm{~m}, 1 \mathrm{H}), 4.14(\mathrm{dd}, J=12.0,4.5 \mathrm{~Hz}, 1 \mathrm{H}), 3.97(\mathrm{dd}, J=11.9,6.0 \mathrm{~Hz}, 1 \mathrm{H}), 2.82(\mathrm{dq}, J=$ 7.2, $5.7 \mathrm{~Hz}, 1 \mathrm{H}), 2.12(\mathrm{~s}, 3 \mathrm{H}), 2.06(\mathrm{~s}, 3 \mathrm{H}), 2.03(\mathrm{~s}, 3 \mathrm{H}), 2.01(\mathrm{~s}, 3 \mathrm{H}), 1.98(\mathrm{~s}, 3 \mathrm{H}), 1.97$ (s, $3 \mathrm{H}$ ), 1.85 (ddd, $J=15.8,10.0,5.21 \mathrm{H}$ ), 1.69 (ddd, $J=15.2,10.7,2.5 \mathrm{~Hz}, 1 \mathrm{H}$ ), 1.05 (d, $J=7.2$ $\mathrm{Hz}, 3 \mathrm{H})$.

${ }^{13}$ C NMR $\left(126 \mathrm{MHz}, \mathrm{CDCl}_{3}\right) \delta 208.7,170.3,170.1,170.1,170.0,169.9,71.2,69.4,68.7$, $67.2,61.8,50.3,30.4,28.9,20.9,20.8,20.8,20.7,20.7,11.2$. 


\section{Structure determination of initial experiment}

Spectra of initial experiment: D-ribose with acetylacetone in the presence of catalytic amounts of pyrrolidine.

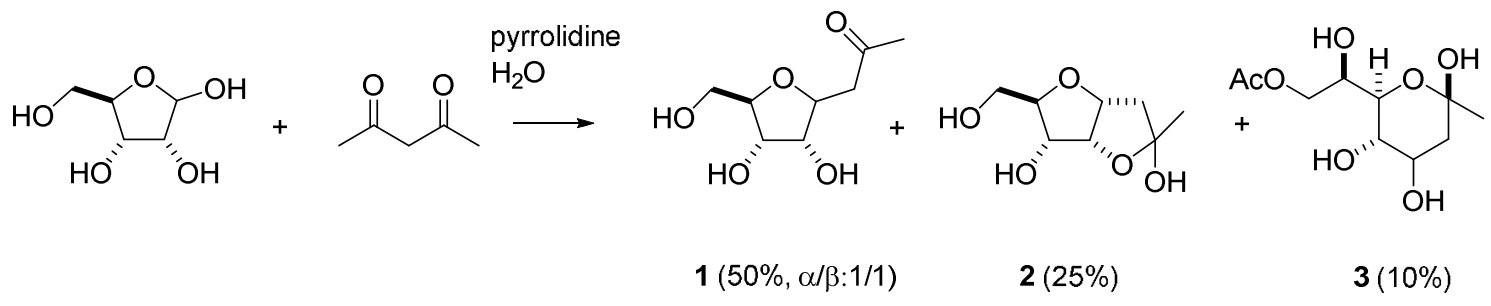

A set of signals for three different C-Glycosides and a chain elongated product (3) was observed. These signals for the two C-glycosides correlate with the spectral data for the $\beta / \alpha-$ furanoid structures of C-ribo-glycoside 1 and the respective $\alpha$-hemiketal structure 2.

For comparison see:

a) Wang, J., Li, Q., Ge, Z., Li, R. Tetrahedron 68, 4, 1315-1320

b) Riemann, I., Fessner, W. D., Papadopoulos, M. A., Knorst, M. Austr. J. Chem. 55, 2, 147154.

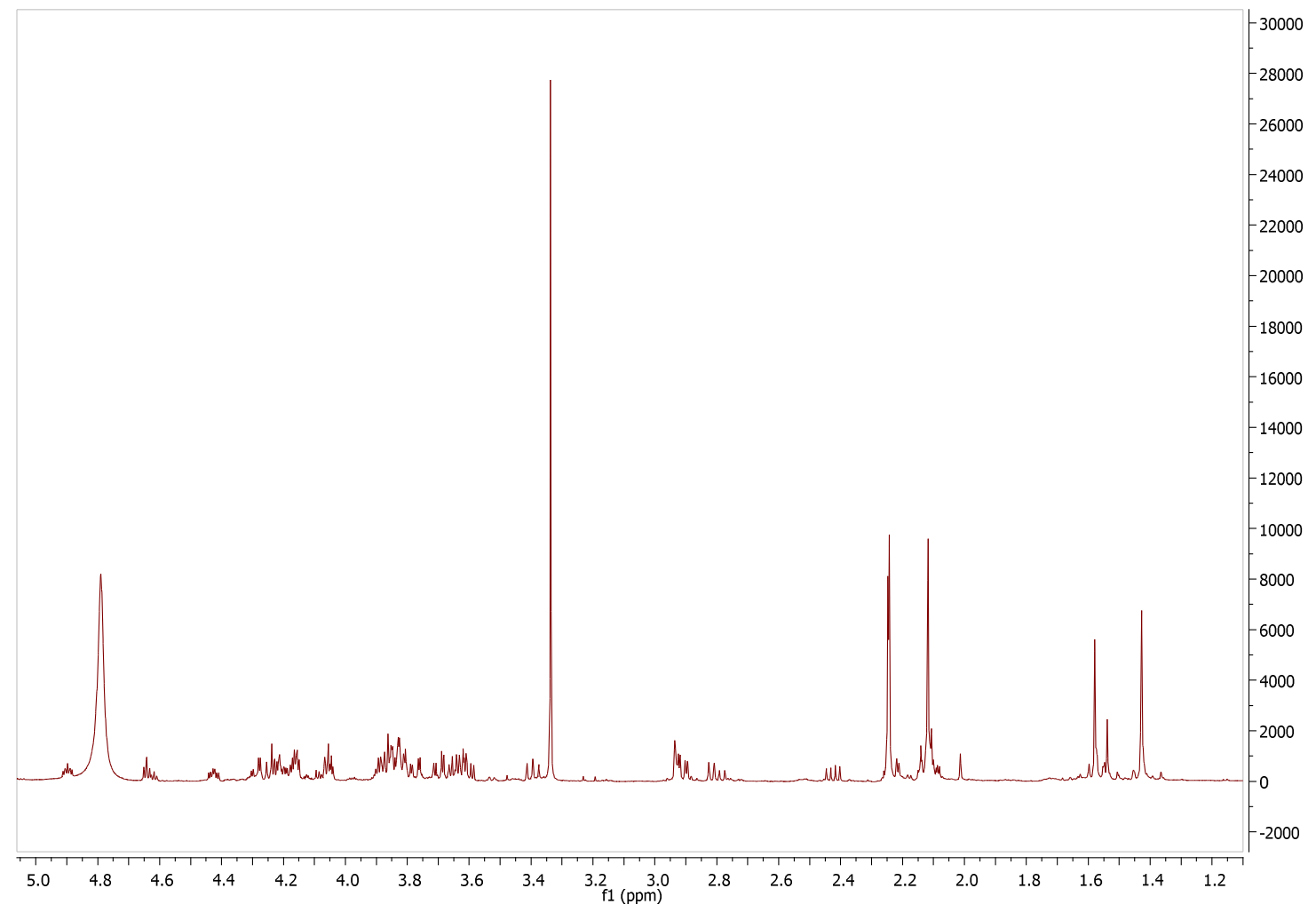




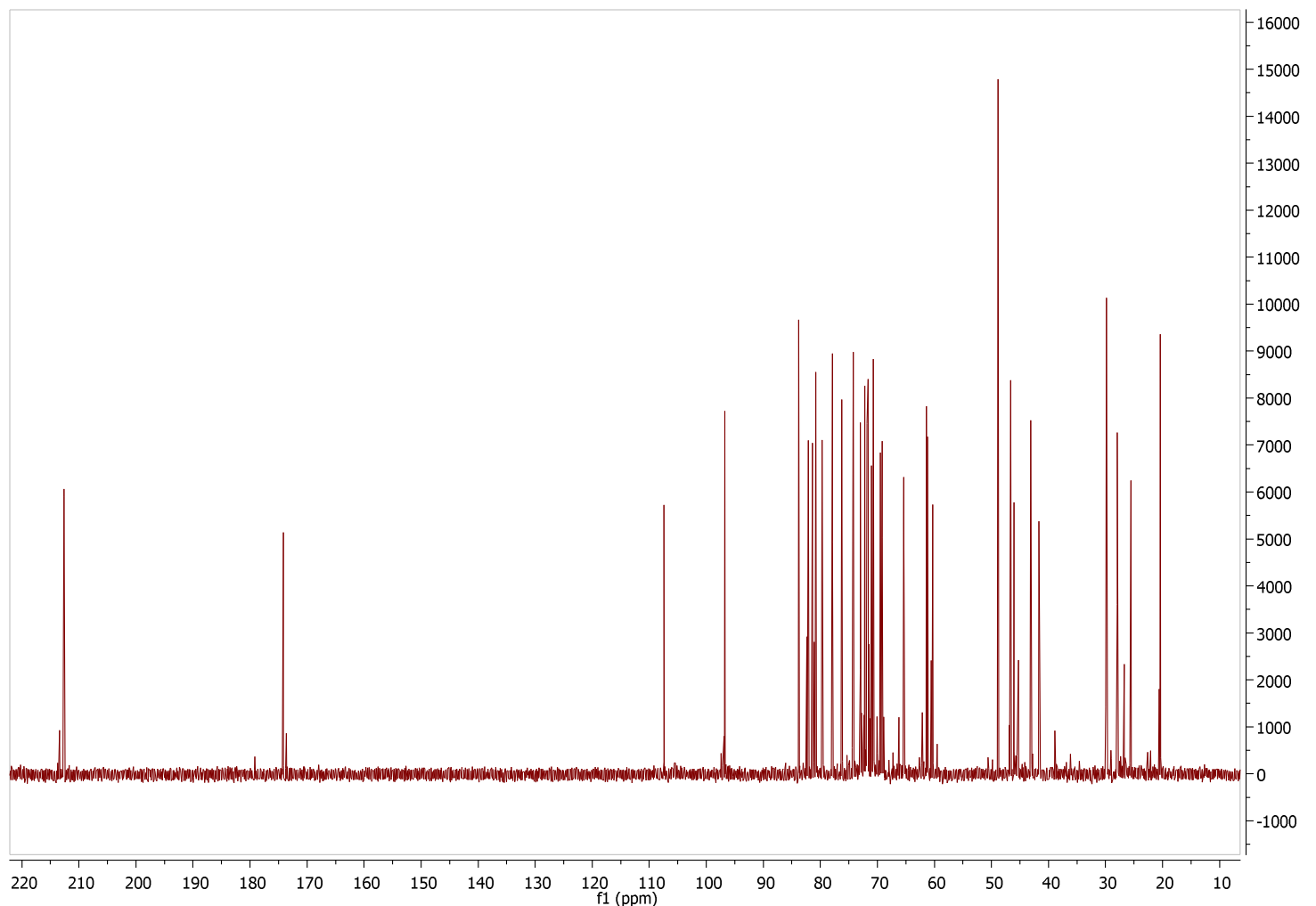




\section{Proof of configuration}

In general the proof of configuration was realized by NMR-experiments of both, the cyclic reaction product and of the peracetylated acyclic structures.

proof of configuration for compound anti-3

Compound anti-3 was observed in the 8-monoacetylated form within an inseparable complex mixture of side products. The signal at $96.8 \mathrm{ppm}\left({ }^{13} \mathrm{C} \mathrm{NMR}\right)$ couples with the neighboring $\mathrm{CH}_{2}$-group at $2.07 \mathrm{ppm}$ and $1.86 \mathrm{ppm}$ (HMBC-experiments). The coupling constants of C3protons with the C4-proton are $3.7 \mathrm{~Hz}$ and $2.9 \mathrm{~Hz}$. Coupling constants of $12.4 \mathrm{~Hz}$ and $5.0 \mathrm{~Hz}$ were detected for the protons at $\mathrm{C} 3$ and $\mathrm{C} 4$ in syn-configured-product 3. A further analysis was not possible due to overlapping signals in all 2D experiments. Purification and characterization of the anti-3 product was carried out by acetylation and isolation as the antiD-ribo-10 product.
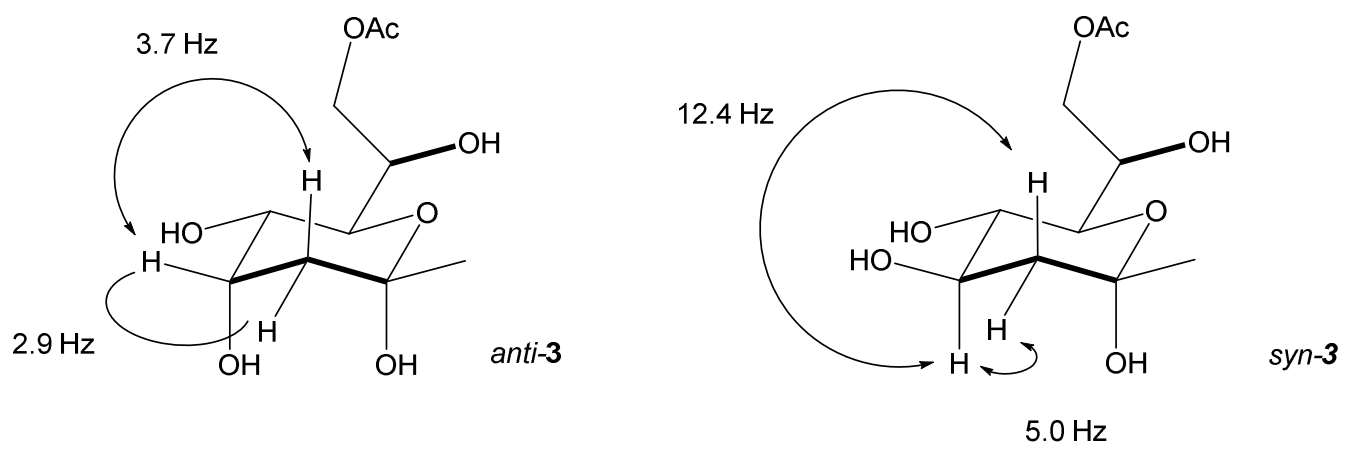

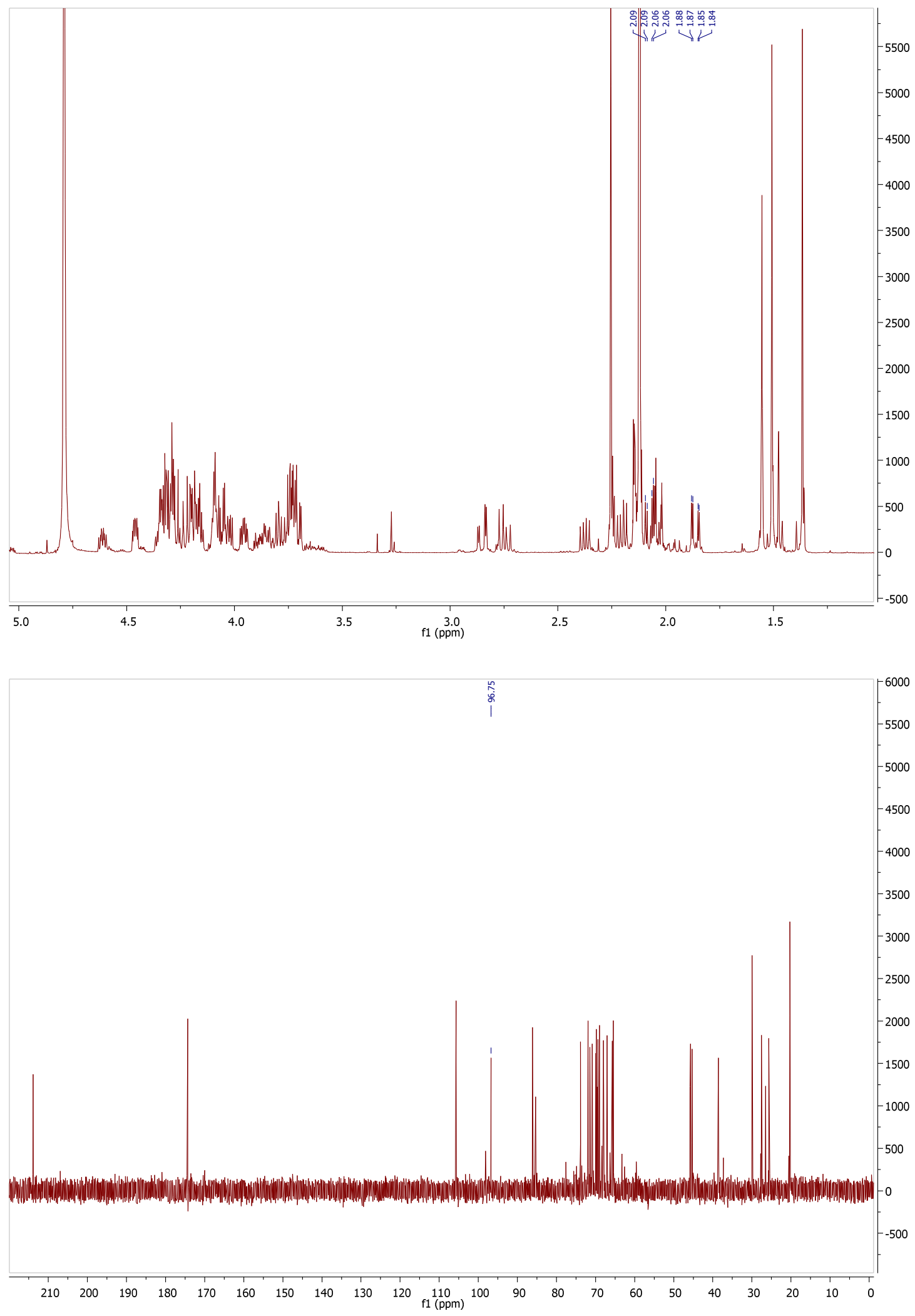


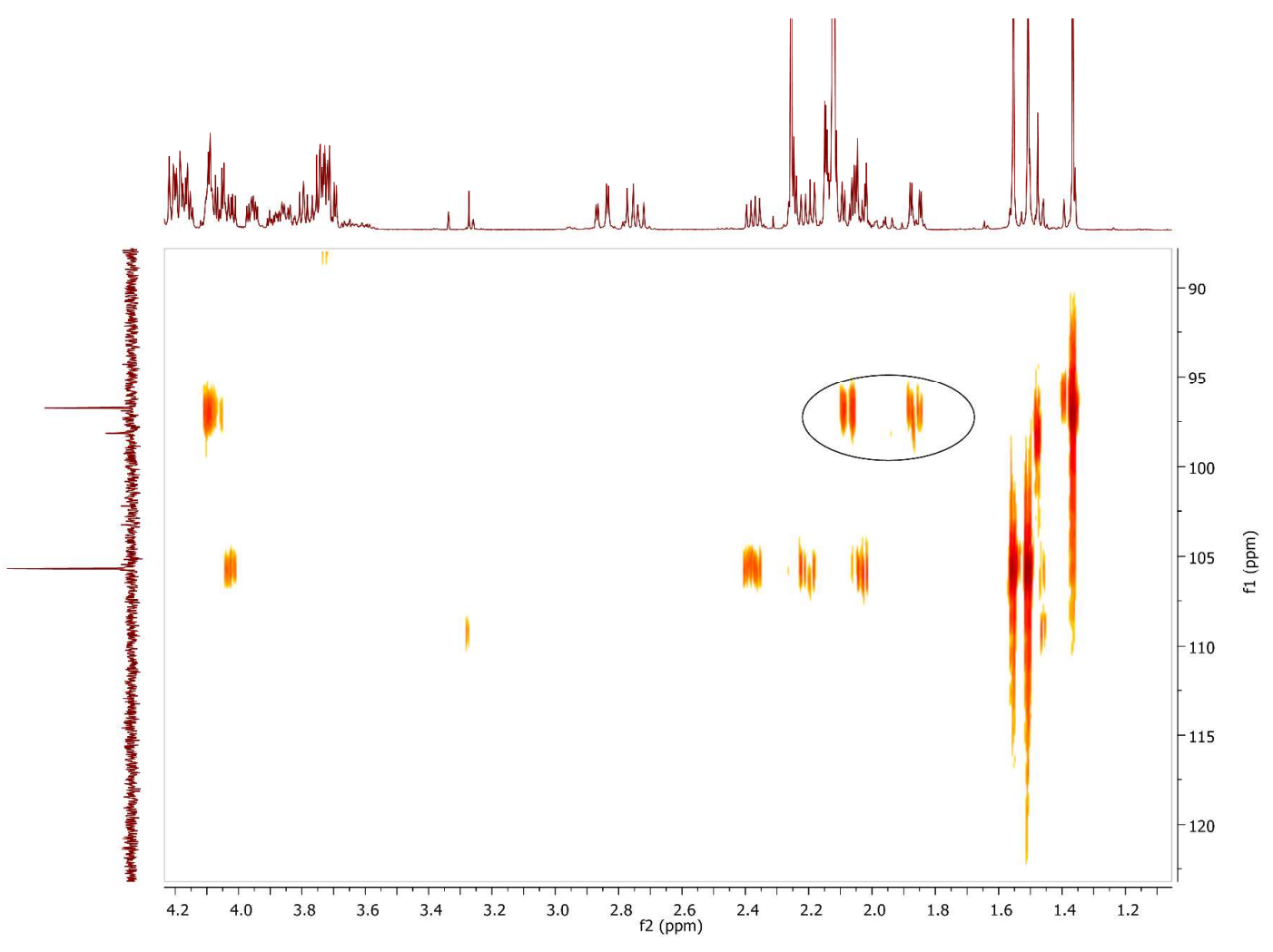

proof of acyl migration

This proof was realized by reactions of D-arabinose with acetylacetone in the presence of catalytic amounts of $\mathrm{N}$-methyl-piperidine.

Small amounts of products with acetyl-groups at secondary alcohols were observed. For example, the signal at $4.94 \mathrm{ppm}$ ( $\mathrm{CH}$-group) couples in $\mathrm{HMBC}$ with the $170 \mathrm{ppm}$ carbon atom. In COSY-experiments this proton couples with a $\mathrm{CH}_{2}$-group (3.67 and $3.80 \mathrm{ppm}$ ) and a $\mathrm{CH}$-group (3.99 ppm). The $\mathrm{CH}$-group (3.99 ppm) couples in HMBC-experiments with a ketalcarbon atom. With this evidence, it can be suggested, that the acetyl-group is located at the C7-position. 


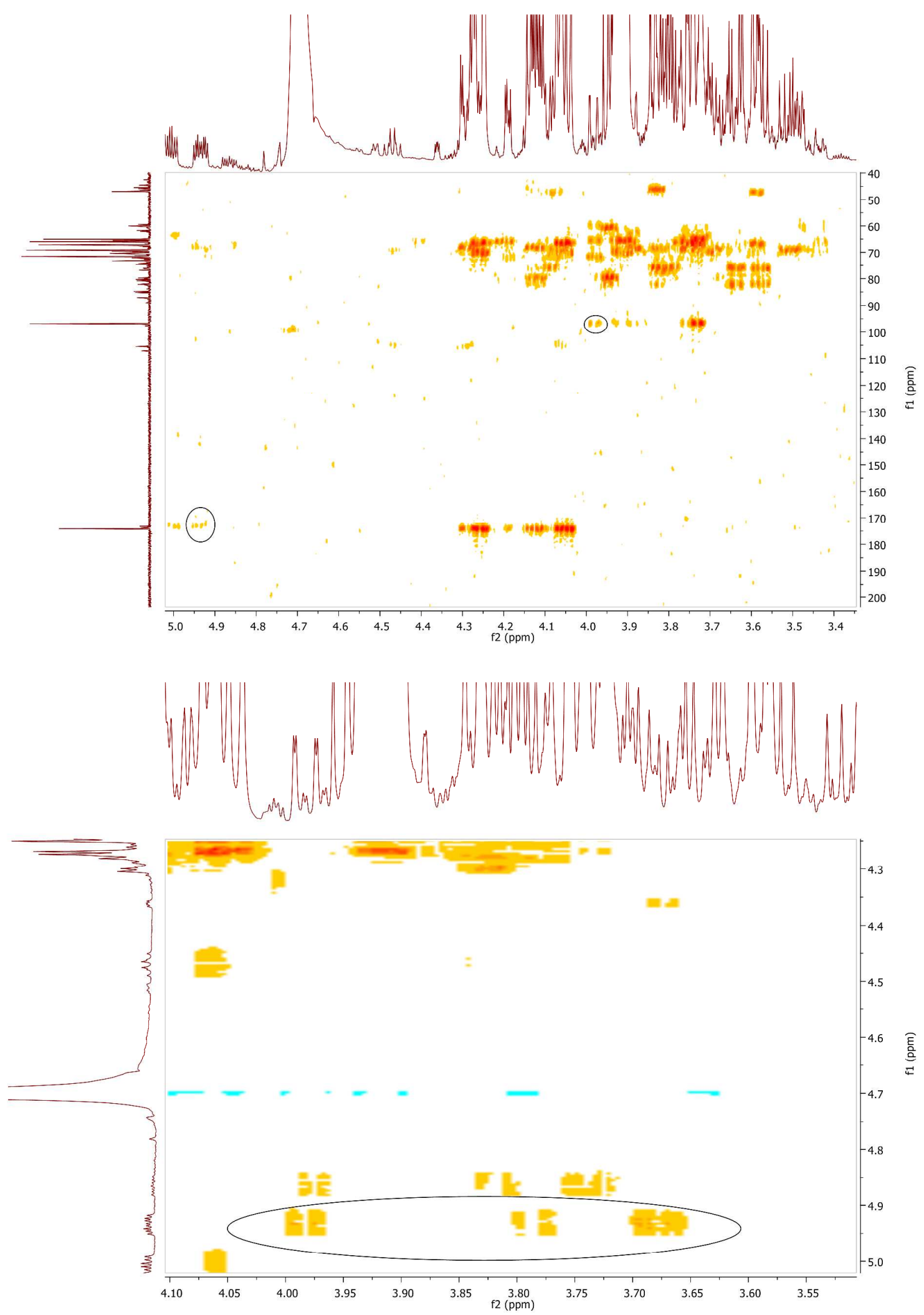




\section{proof of configuration for D-manno-22}

In the literature the reaction of D-mannose with acetylacetone in the presence of aqueous $\mathrm{NaHCO}_{3}$ as base is described by Wang, Junfeng, et al. Tetrahedron 2012, 68, 1315-1320. The product of this reaction is described as the structure $\mathbf{2 c}$. A more convenient illustration of the ketal moiety is given by structure $\mathbf{A}$

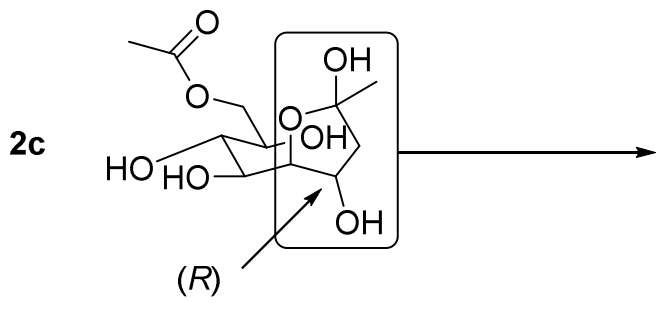

$(R)$

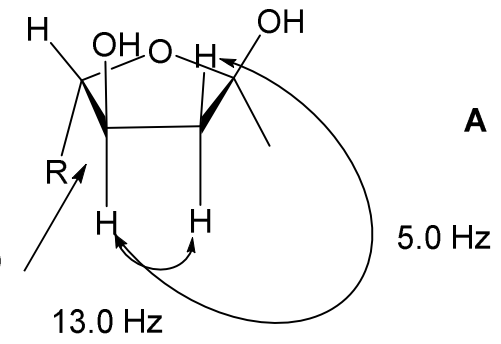

The pseudoanomeric carbon atom should be $(R)$-configured by this representation.

The spectral data in the literature are as follows:

${ }^{1} \mathrm{H}$ NMR $\left(500 \mathrm{MHz}, \mathrm{D}_{2} \mathrm{O}\right) \delta 4.40$ (dd, $\left.J=12.0,2.5 \mathrm{~Hz}, 1 \mathrm{H}\right), 4.28(\mathrm{dd}, J=12.0,5.5 \mathrm{~Hz}, 1 \mathrm{H})$, $3.99-3.86(\mathrm{~m}, 4 \mathrm{H}), 3.47(\mathrm{t}, J=9.5 \mathrm{~Hz}, 1 \mathrm{H}), 2.15(\mathrm{dd}, J=13.0,5.0 \mathrm{~Hz}, 1 \mathrm{H}), 2.13(\mathrm{~s}, 3 \mathrm{H})$, $1.59(\mathrm{~s}, J=13.0 \mathrm{~Hz}, 1 \mathrm{H}), 1.44(\mathrm{~s}, 3 \mathrm{H})$

${ }^{13}$ C NMR $\left(126 \mathrm{MHz}, \mathrm{D}_{2} \mathrm{O}\right) \delta$ 175.26, 97.56, 71.23, 71.17, 70.02, 68.96, 68.41, 67.12, 42.48, $28.71,21.01$.

In experiments of acetylacetone with D-mannose we isolated a product with the same spectral data as described above.

The configuration at the pseudoanomeric carbon atom was determined as a $(S)$-configured one by several NMR-experiments (structure B).
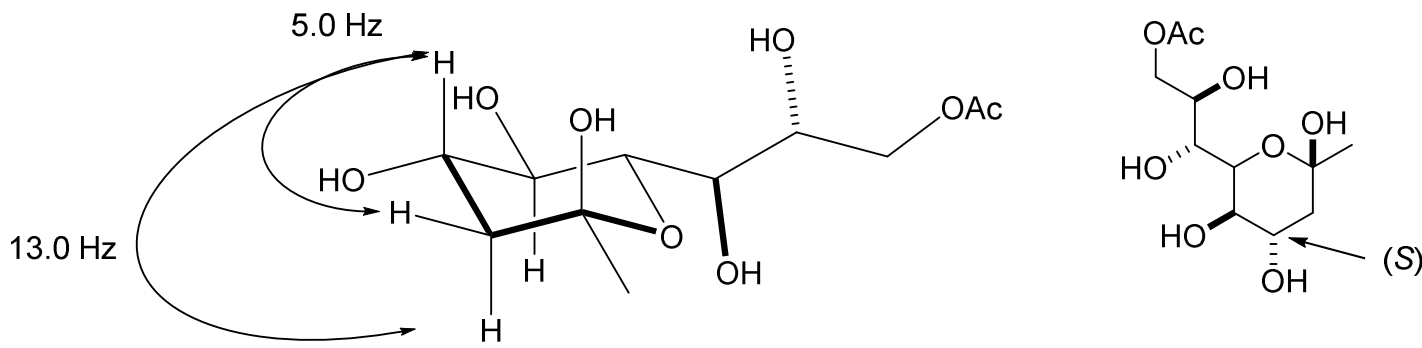

There is a contradiction to the results described above. The pseudoanomeric carbon atom in structure $\mathbf{A}$ is $(R)$-configured, whereas the pseudoanomeric carbon atom in structure $\mathbf{B}$ is (S)-configured. The coupling constants found in the ${ }^{1} \mathrm{H}$ NMR spectra explain both structures, A or B. 
The argumentation to prove the structure is as follows:

The coupling constants are indicated in both structures, A and B. In structure A the dihedral angles of the $\mathrm{CH}_{2}$-group and the pseudoanomeric proton are $0^{\circ}$ and $100^{\circ}$ respectively. This means, the proton with the $0^{\circ}$ angle should have the higher coupling constant. It is positioned below the furan plane. The C2-proton is also axial, it is positioned above the furan plane. So it should not be possible for them, to couple with each other in a NOESYexperiment.

In contrast, in structure $\mathbf{B}$ one of the $\mathrm{CH}_{2}$-protons (with the high-coupling constant), as well as the C2-proton (triplet) are facing the same direction and should give a discrete signal in a NOESY-experiment.

This is what we detected. Below the NOESY-NMR spectrum of syn-D-manno-22 (cyclic and acyclic) is given. This molecule was obtained directly from acetylating the molecule in question. For ${ }^{1} \mathrm{H}$ and ${ }^{13} \mathrm{C}$ see "Copy of NMR Spectra". Note, that the NMR-spectra represents a mixture of the cyclic and the acyclic structure.

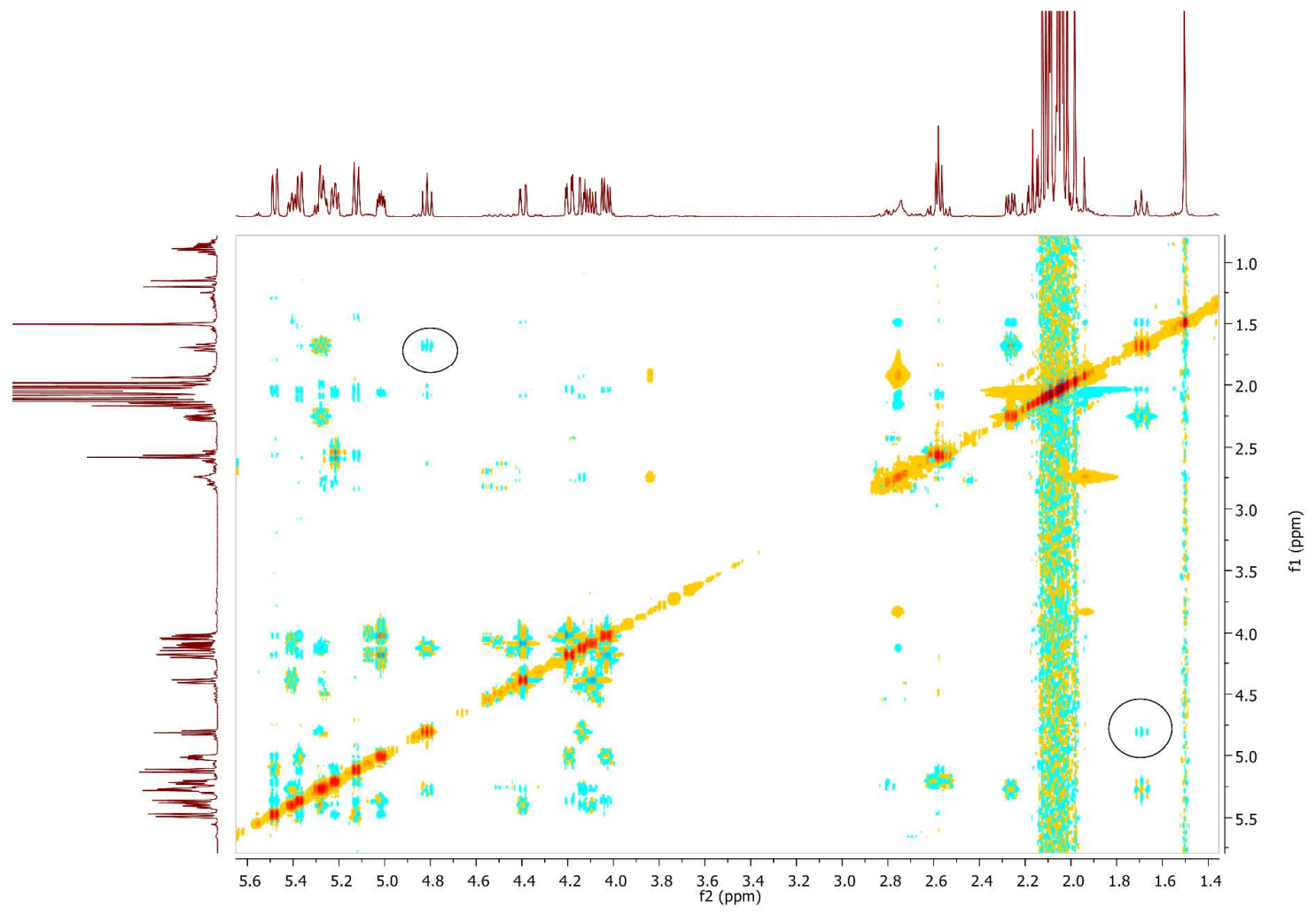

A NOESY-coupling can be found between the $1.67 \mathrm{ppm}$ proton with the high coupling constant and the triplet at $4.79 \mathrm{ppm}$, thus proving structure $\mathbf{B}$ to be the correct one. Just for further prove, as a mixture of two products can be hard to differentiate, the HMBC and HSQC spectra are shown below. 


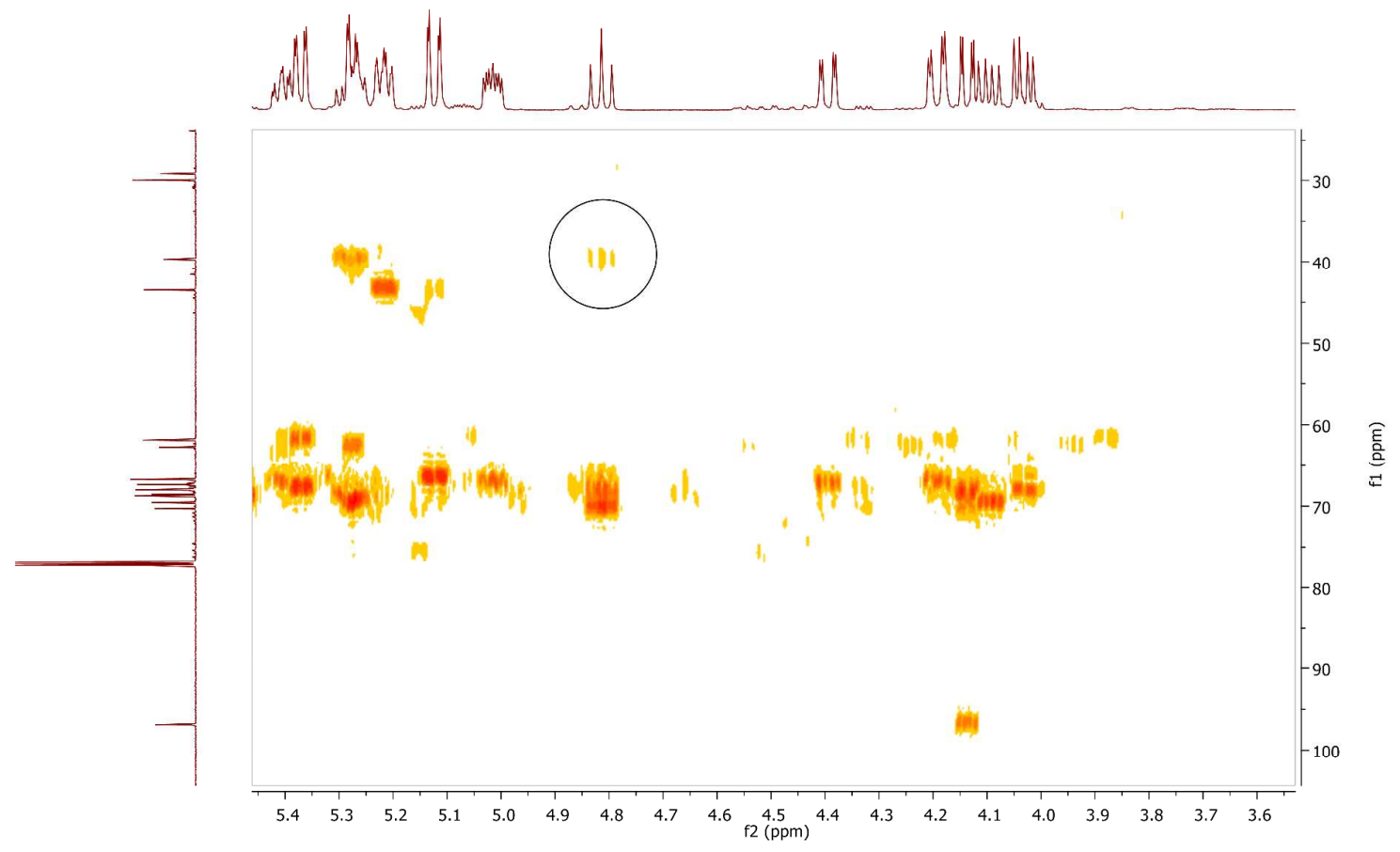

A coupling of the $4.79 \mathrm{ppm}$ proton signal with the $40.0 \mathrm{ppm}$ carbon signal was observed. In an HSQC-experiment this signal corresponds directly with the $1.67 \mathrm{ppm}$ proton.

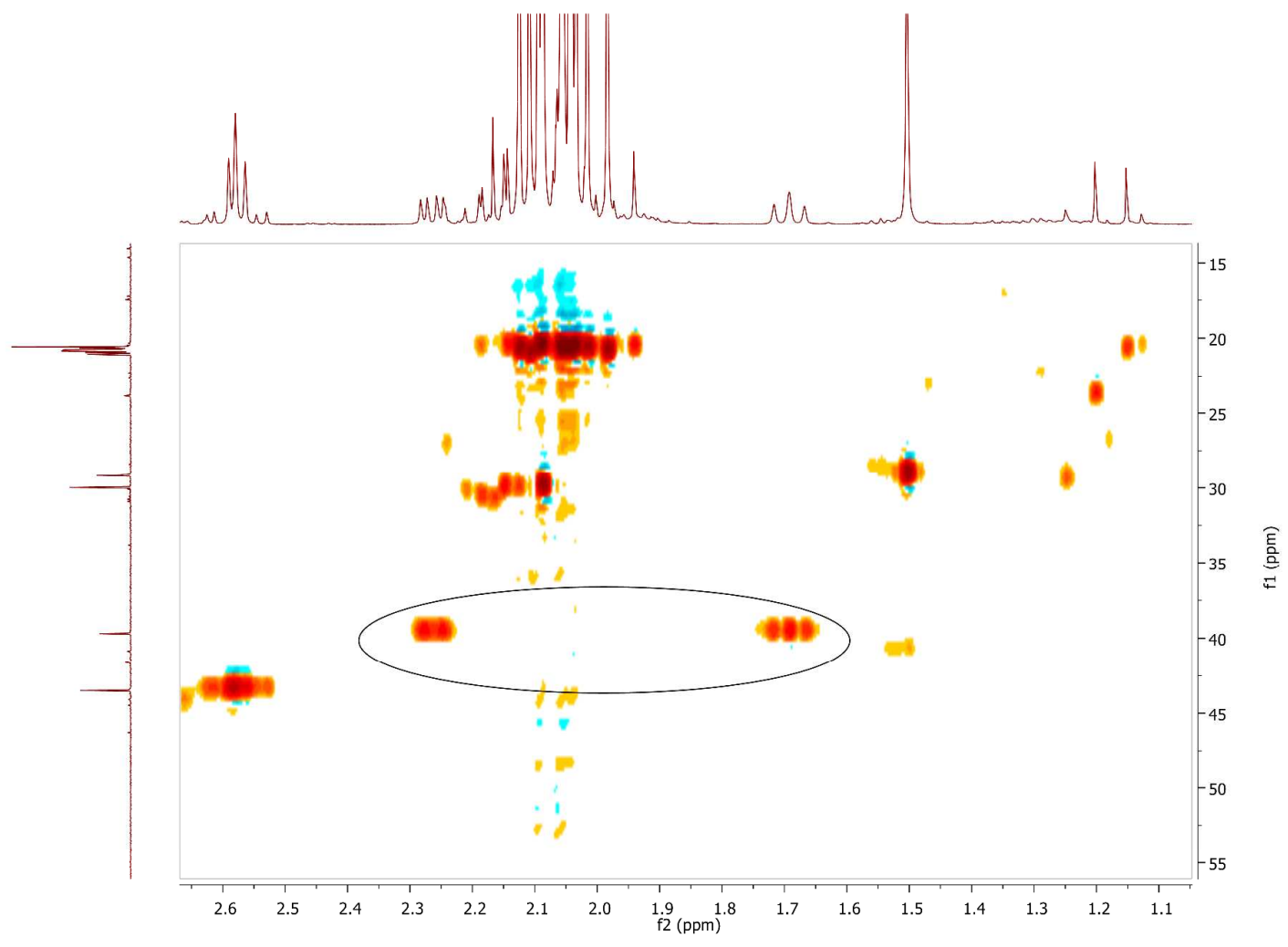




\section{Conformation analysis and proof of configuration of D-deoxygalacto-25}

conformers with 3.4-syn-configuration<smiles>OCC(O)C(O)C1CC2OC3OC2CC3C1O</smiles><smiles>CC1CC2(O)OC(C(O)C(O)CO)CC1C(O)C2C</smiles><smiles>CC1C(O)CC(C(O)C(O)CO)OC(C)(O)C1C</smiles><smiles>CC1CC2(O)CC(C(O)C(O)CO)CC1CO2</smiles><smiles>CC(=O)C(C)C(O)C[C@@H](O)C(O)C(O)CO</smiles><smiles>CC(=O)[C@H](C)[C@H](O)C[C@@H](O)C(O)C(O)CO</smiles>

conformers with 3.4-anti-configuration<smiles>CC1CC2C(O)OC(C(O)C(O)CO)CC1C2O</smiles><smiles>CC1C2OC(C(O)C(O)CO)CC(O)(C2C)C1O</smiles><smiles>CC1C2CC(C(O)C(O)CO)OC(C)(O)C1O2</smiles><smiles>CC1C(O)CC(C(O)C(O)CO)OC1(C)O</smiles><smiles>C=C</smiles><smiles>CC(=O)C(C)[C@H](O)C[C@@H](O)[C@H](O)C(O)CO</smiles><smiles>CC(=O)[C@H](C)CC(O)C(O)C(O)CO</smiles> 


\section{$\underline{3.4-s y n \text {-configured } 25}$}

C7: 5.32 , dd $(4.7,9.3)$, acetone- $d_{6}$

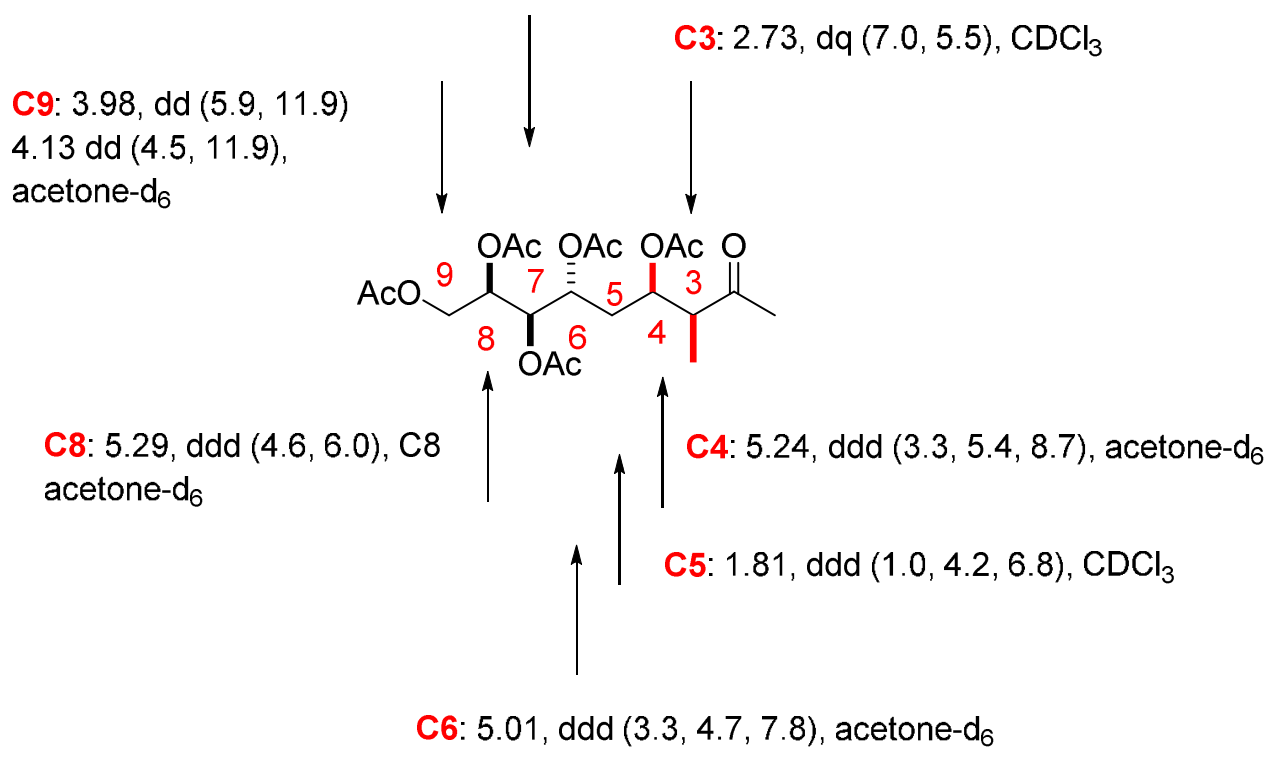

\section{4-anti-configured 25}

C9: 3.98 , dd $(6.1,12.0)$

$4.13 \mathrm{dd}(4.1,11.9)$, acetone- $\mathrm{d}_{6}$

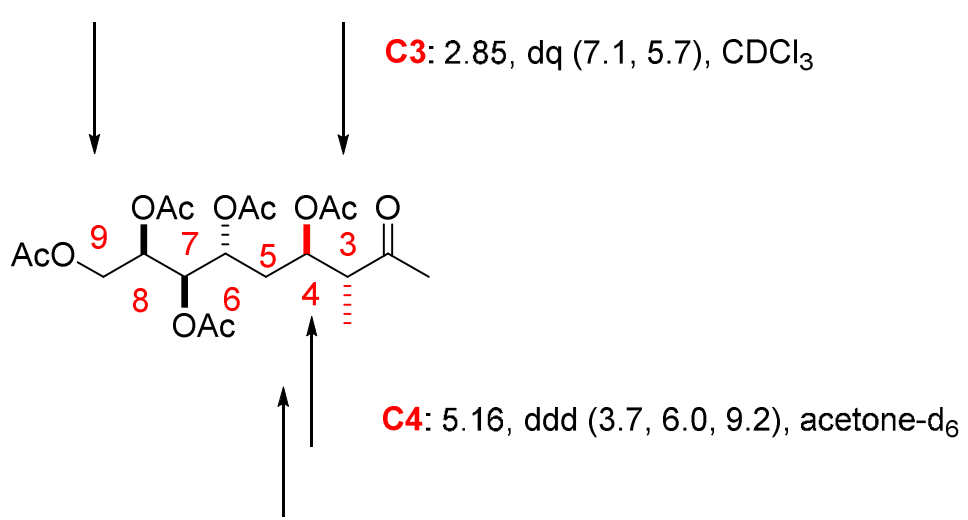

C5: 1.79 , ddd $(2.5,10.7,15.2)$ $1.85 \mathrm{ddd}(5.2,10.0,15.8), \mathrm{CDCl}_{3}$

The differences of $5.5 \mathrm{~Hz}$ (2.73 ppm, C3/C4, syn-25) and $5.7 \mathrm{~Hz}(2.85 \mathrm{ppm}, \mathrm{C} 3 / \mathrm{C} 4$, anti-25) are too small for an exact assignment of configuration. However, the chemical shift of the protons at C3, C4 and the Me-group at C3 gives evidence for syn- and anti-configuration. Low-field shift for the doublet of the Me-group at C3:

1.05, d (J=7.2 Hz) $\rightarrow$ anti 
$1.02, \mathrm{~d}(\mathrm{~J}=7.2 \mathrm{~Hz}) \rightarrow$ syn

proton at C3: 2.85, dq $\rightarrow$ anti; $2.73, \mathrm{dq} \rightarrow$ syn

proton at C4: 5.16, ddd $\rightarrow$ anti; 5.22 , dq $\rightarrow$ syn

Next, a NOE-experiment and the analysis of the coupling constants of the cyclic structure gives more evidence for the 3.4-syn-configuration:

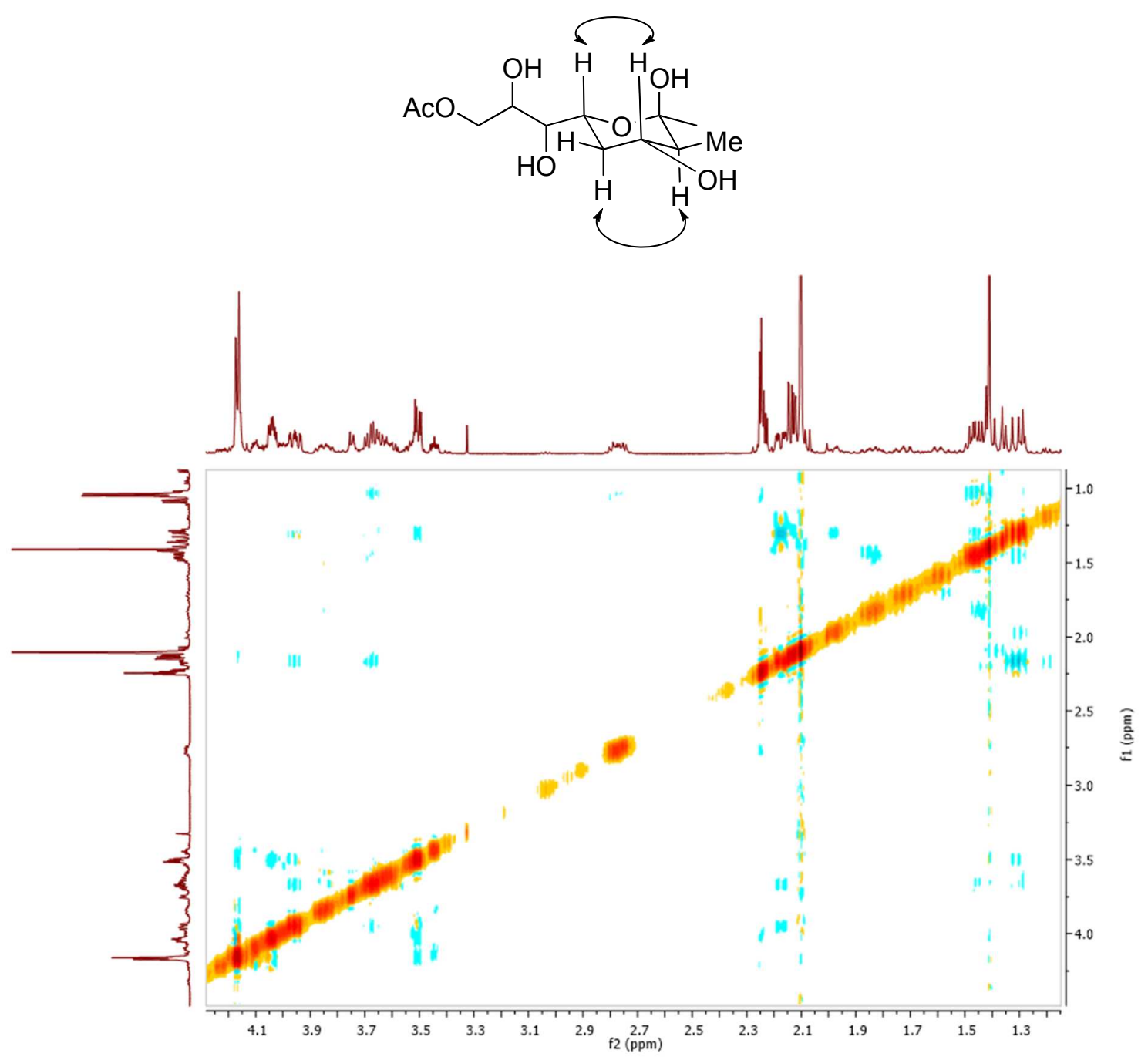


Support for these observations and considerations is given by the analysis of the coupling constants of protons in the cyclic hemiketal structure.

The high coupling constants of appr. $11 \mathrm{~Hz}$ indicate a dihedral angle of appr. $180^{\circ}$ between protons of C3 / C4 and C4 / C5. This value gives evidence for the 3.4-diequatorial configuration in the cyclic structure and thus for the 3.4-syn-configuration in the acyclic structure 25.

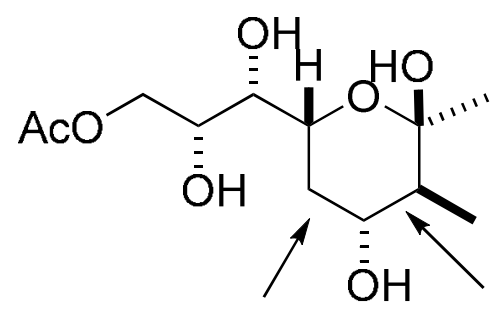

$11 \mathrm{~Hz}$

$11 \mathrm{~Hz}$

\section{Copies of NMR-spectra}


(R)-2-hydroxy-2-((2R,3S,4R,6S)-3,4,6-trihydroxy-6-methyltetrahydro-2H-pyran-2-yl)ethyl acetate syn-3
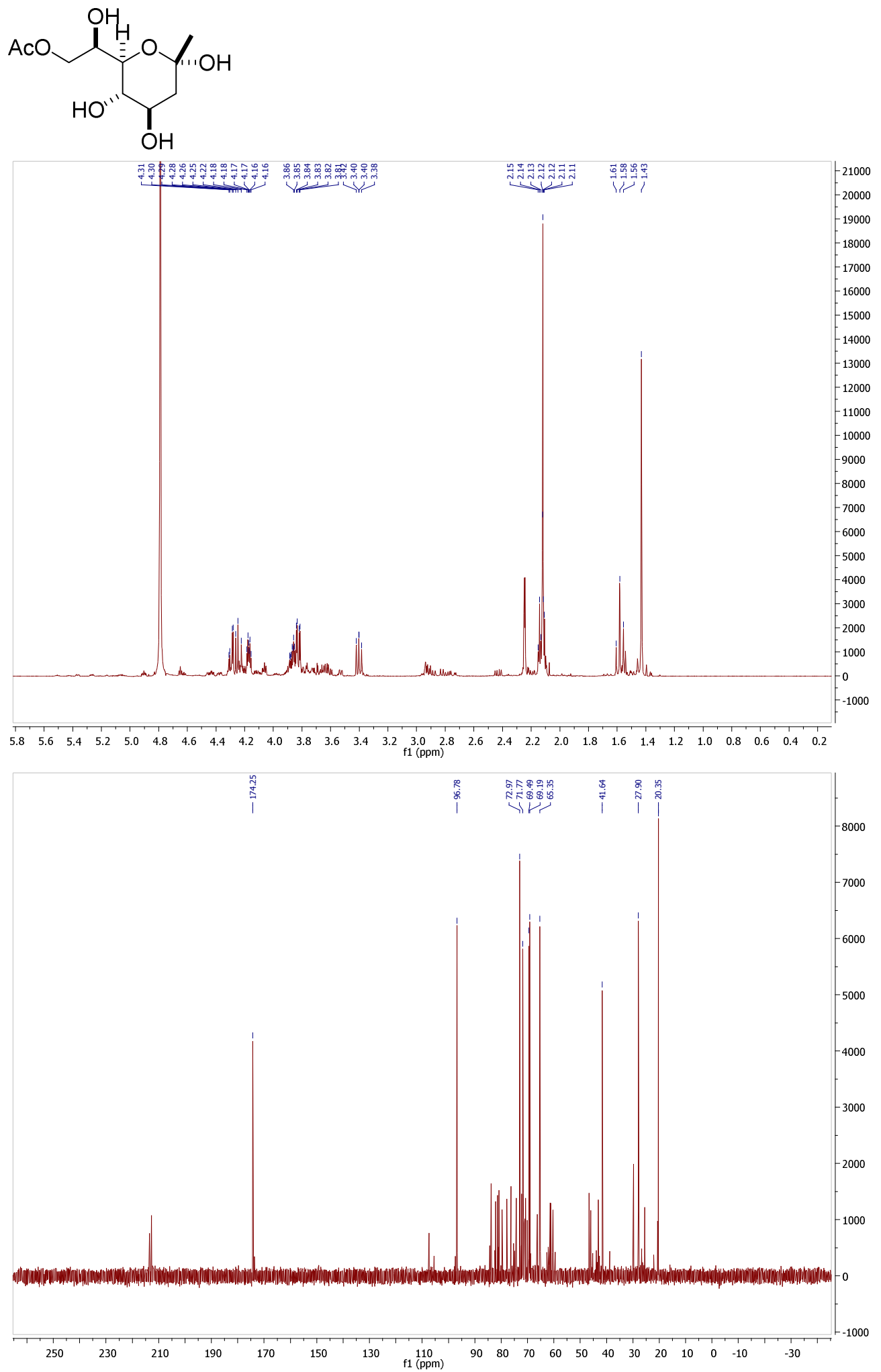

(2R,3R,4S,5S)-7-oxooctane-1,2,3,4,5-pentayl pentaacetate anti-D-ribo-10 


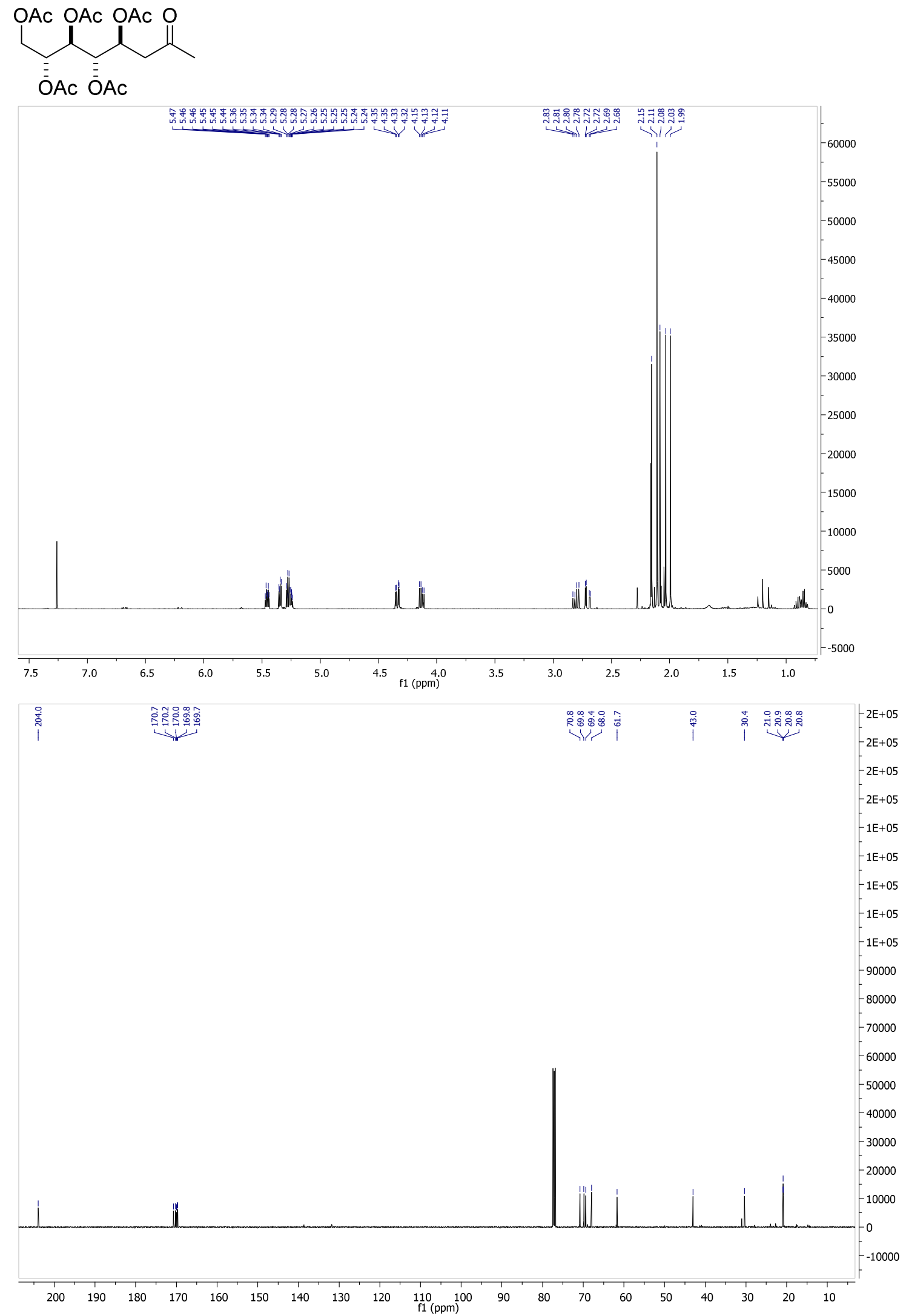

(2R,3R,4S,5R)-7-oxooctane-1,2,3,4,5-pentayl pentaacetate syn-D-ribo-10 + (2R,3R,4S,5S)-2-(acetoxymethyl)-5-(2-oxopropyl)tetrahydrofuran-3,4-diyl diacetate 


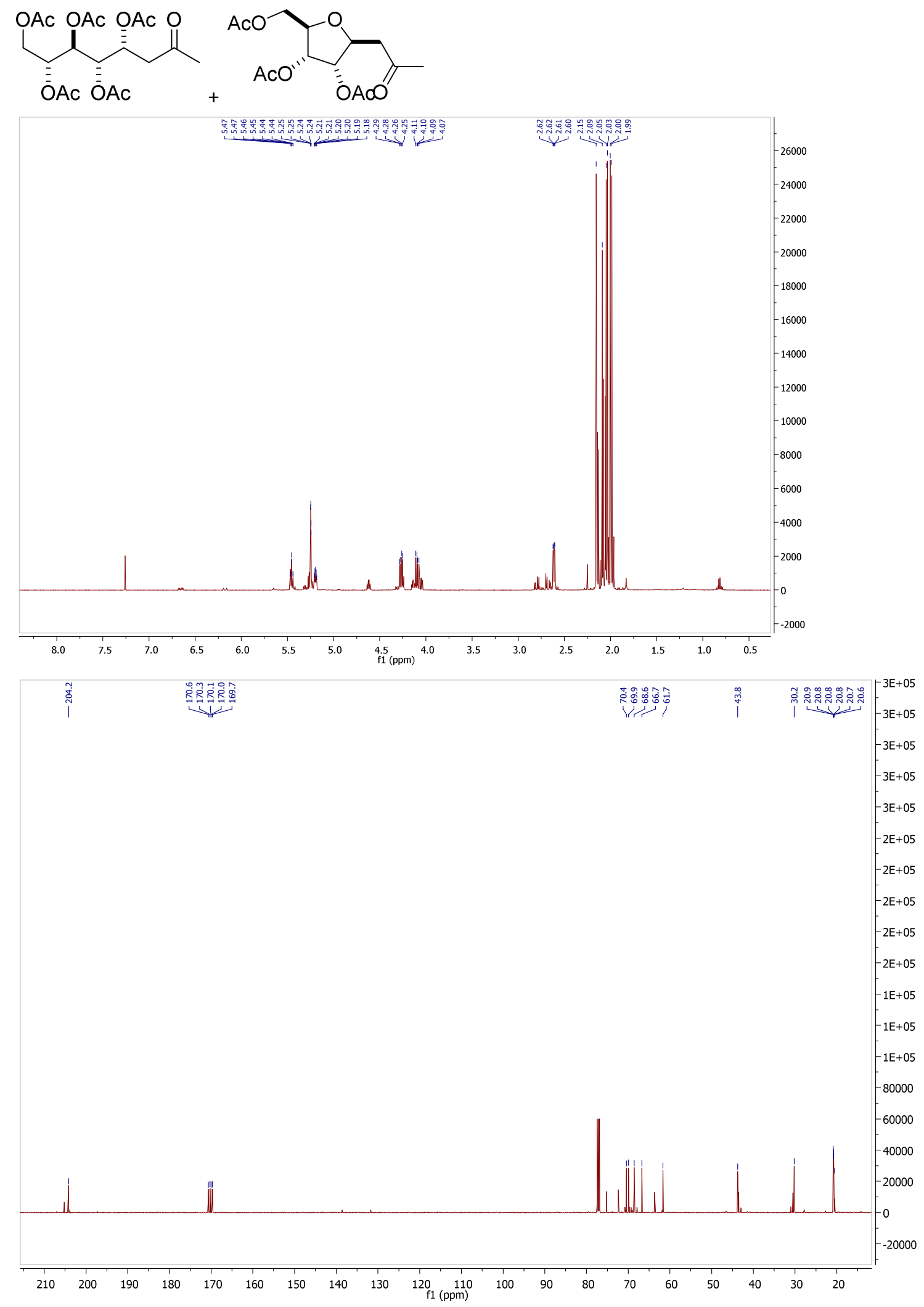

(2S,3S,4R,5R)-7-oxooctane-1,2,3,4,5-pentayl pentaacetate anti-ent-10 


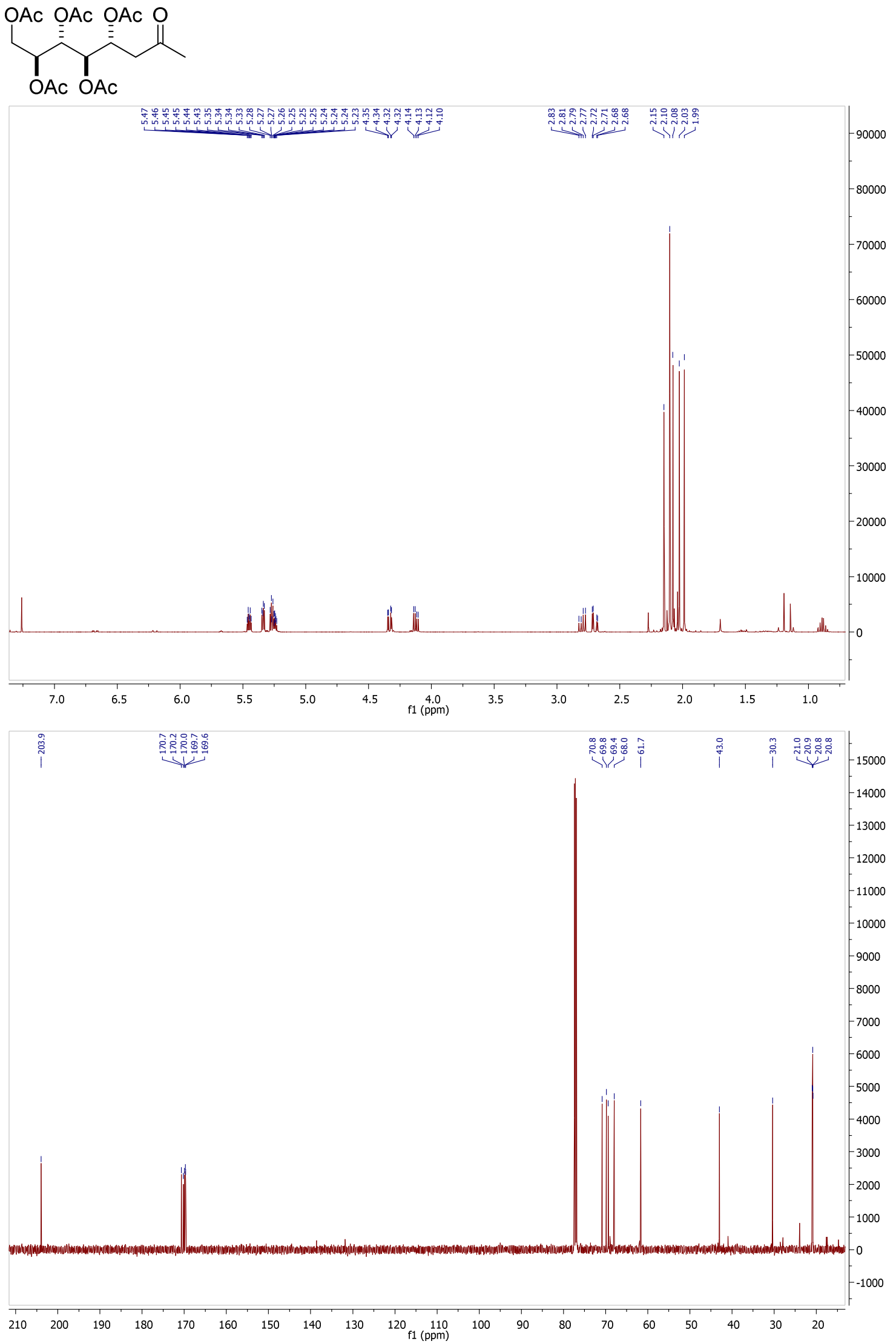

(2S,3S,4R,5S)-7-oxooctane-1,2,3,4,5-pentayl pentaacetate syn-ent-10 

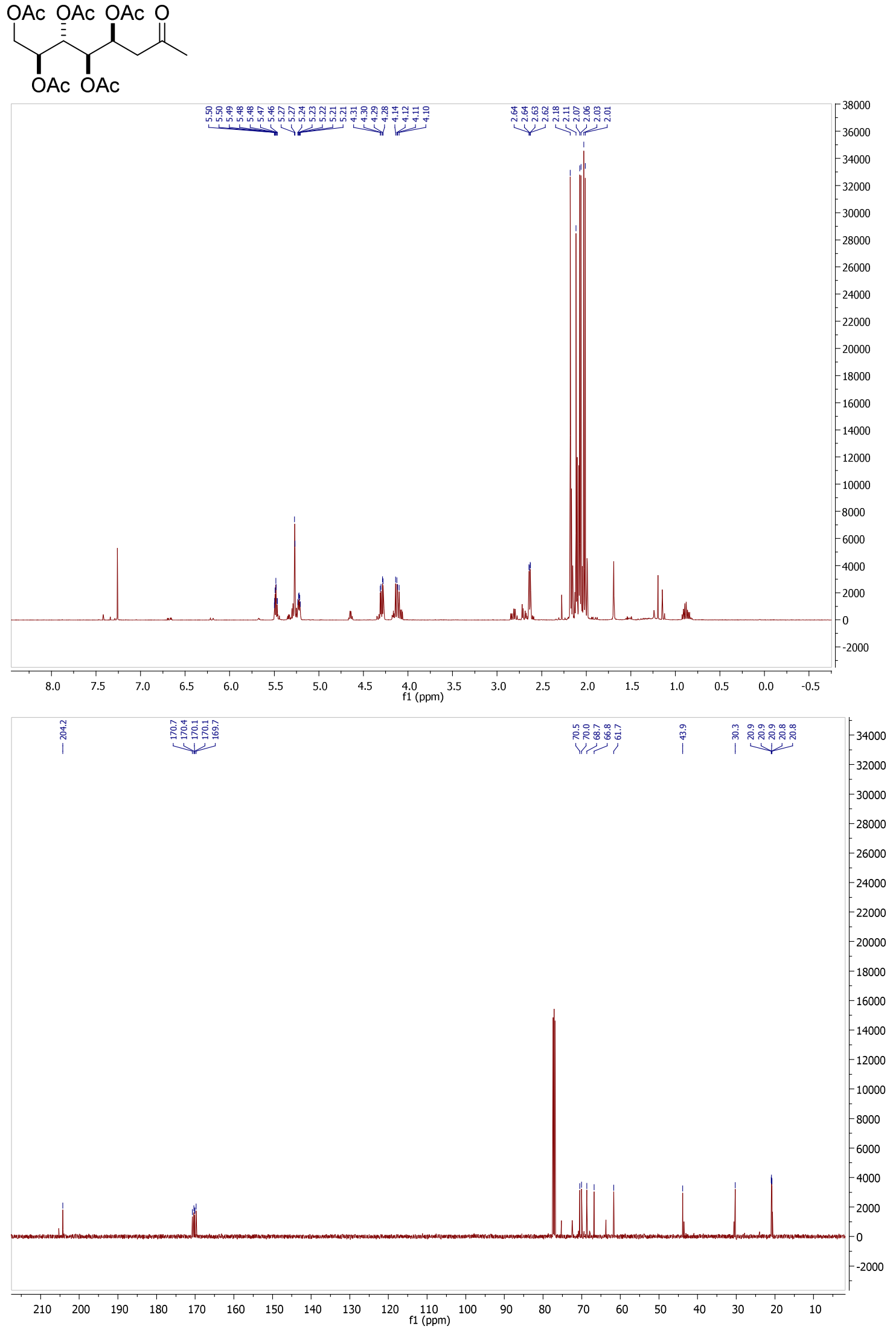

(R)-2-hydroxy-2-((2R,3R,4R,6S)-3,4,6-trihydroxy-6-methyltetrahydro-2H-pyran-2-yl)ethyl acetate 
<smiles>CC(=O)OCC(O)[C@H]1O[C@](C)(O)CC(O)[C@H]1O</smiles>
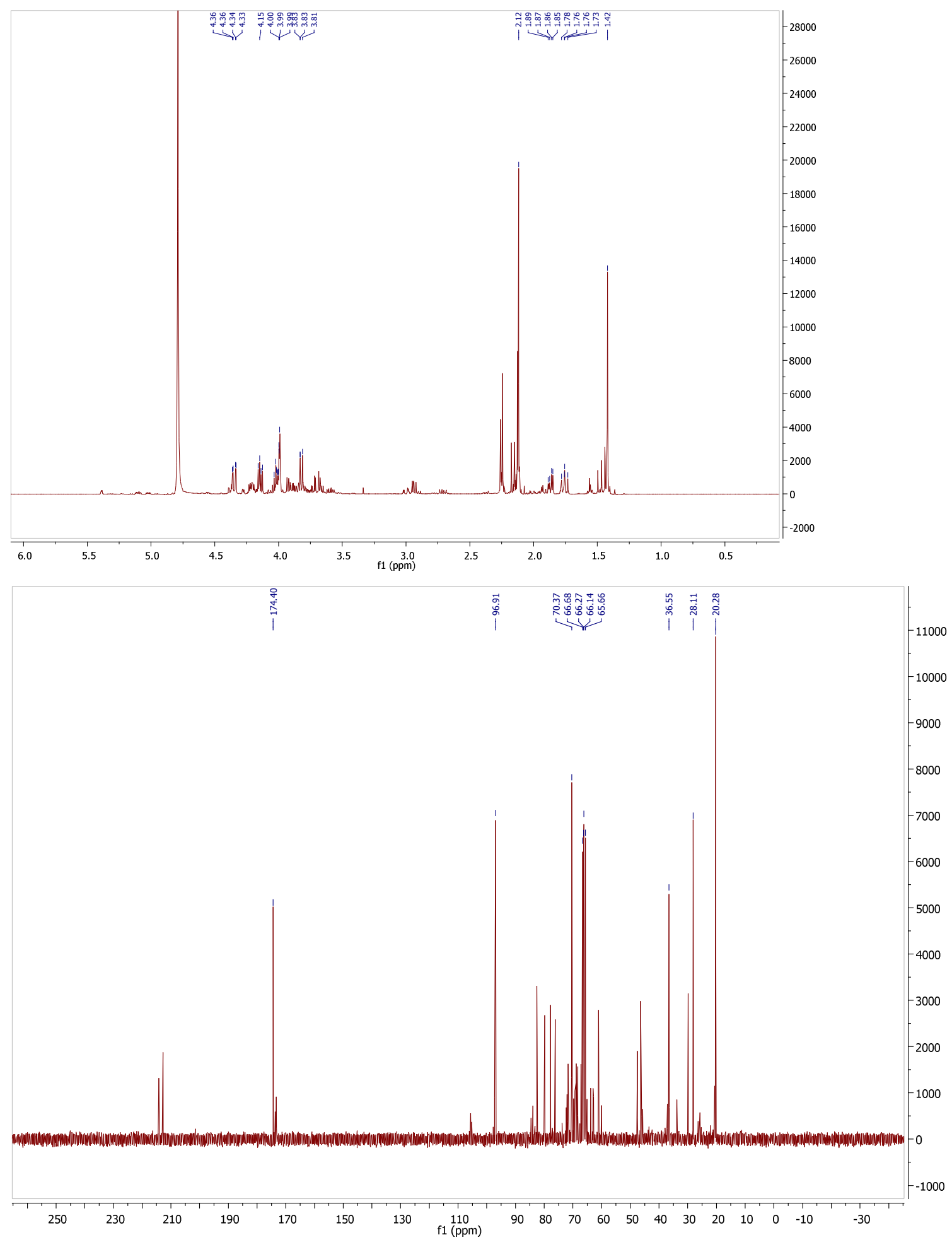

(2R,3R,4R,5R)-7-oxooctane-1,2,3,4,5-pentayl pentaacetate anti-D-ara-11 

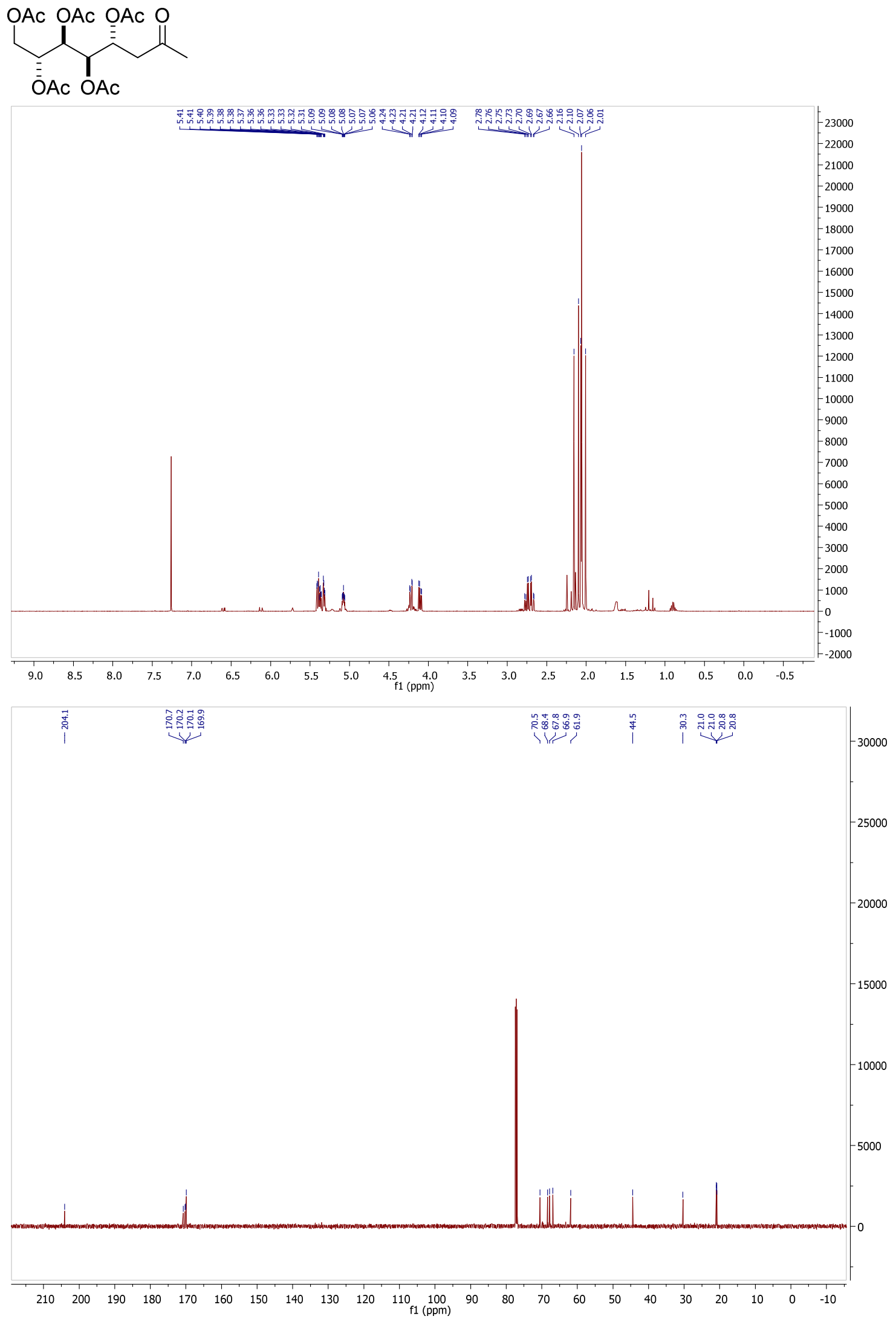

(2S,3S,4S,5S)-7-oxooctane-1,2,3,4,5-pentayl pentaacetate anti-ent-11 


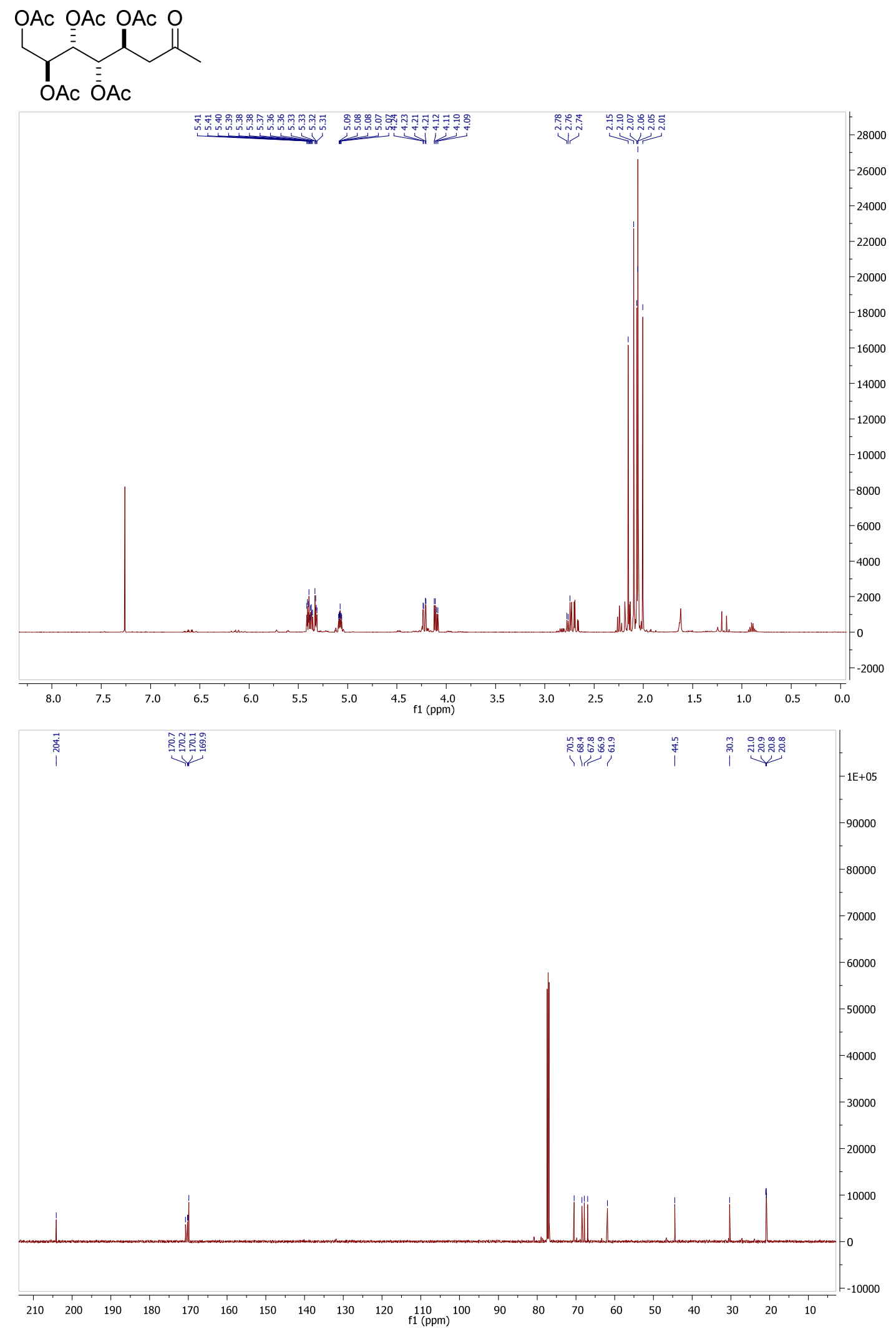

(2R,3S,4R,5R)-7-oxooctane-1,2,3,4,5-pentayl pentaacetate anti-D-lyxo-12 


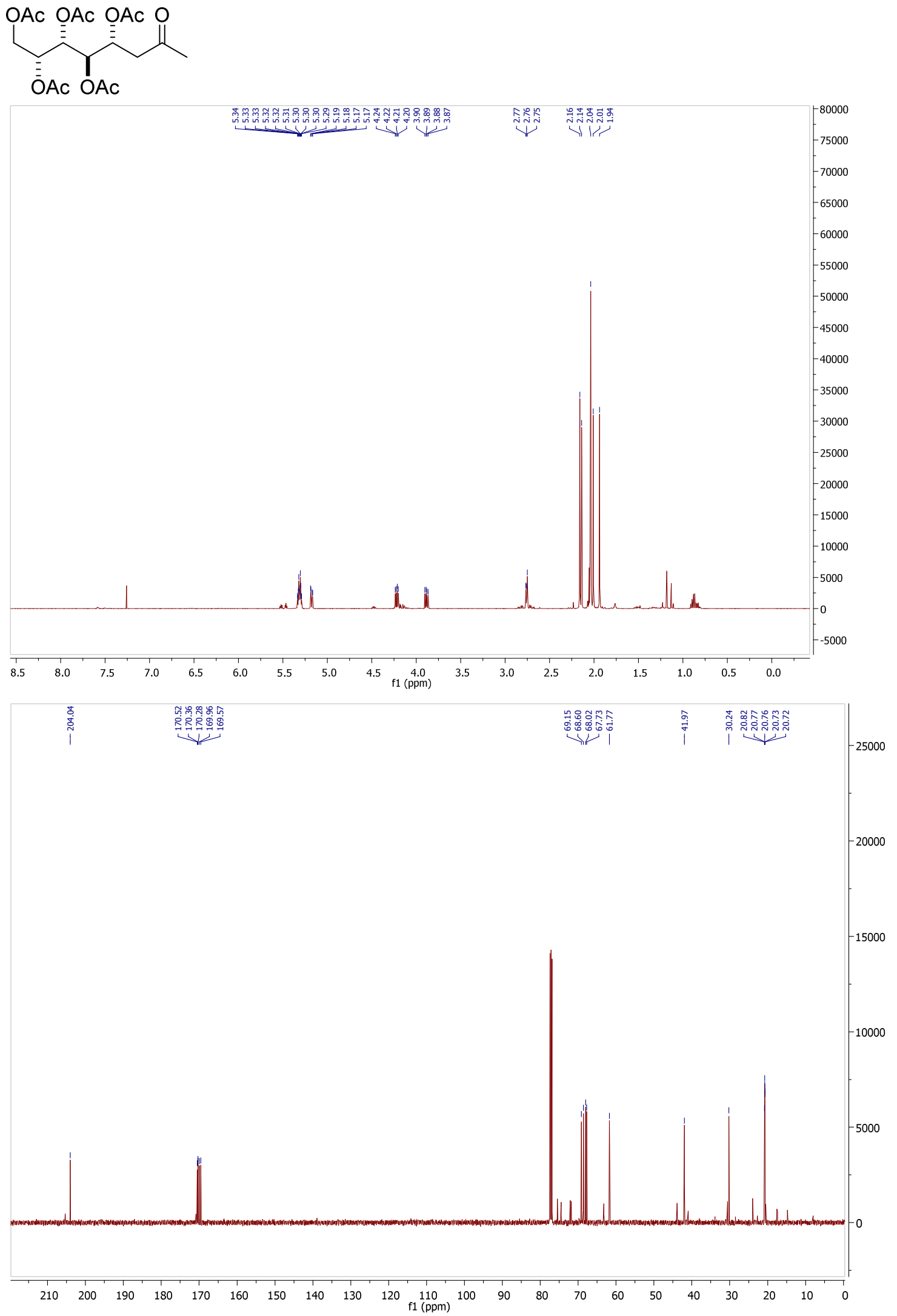

(2R,3S,4R,5S)-7-oxooctane-1,2,3,4,5-pentayl pentaacetate syn-D-lyxo-12 

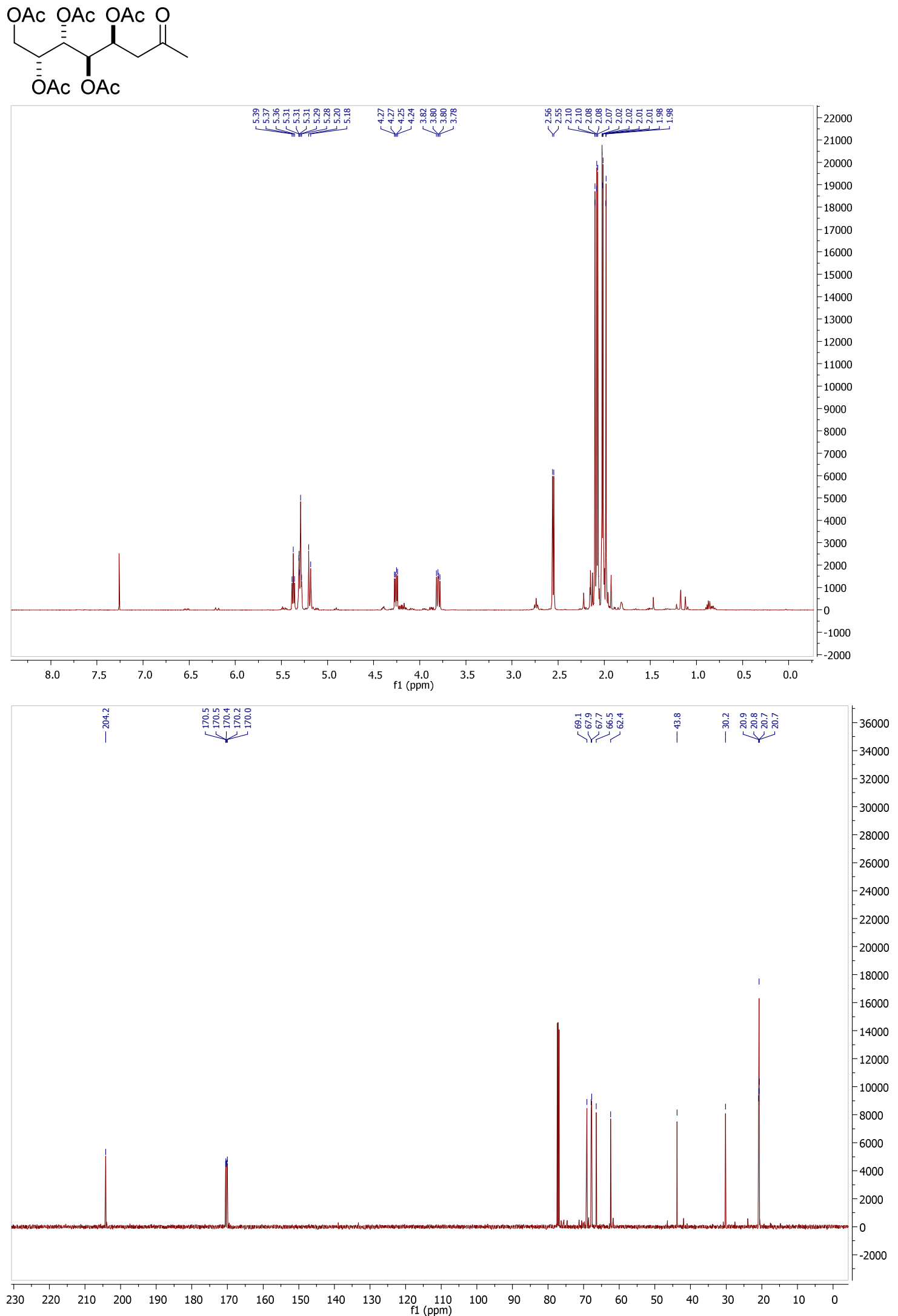
(R)-2-hydroxy-2-((2S,3S,4S,6R)-3,4,6-trihydroxy-6-methyltetrahydro-2H-pyran-2-yl)ethyl acetate<smiles>CC(=O)OCC(O)C1(C)O[C@](C)(O)C[C@H](O)[C@@H]1O</smiles>
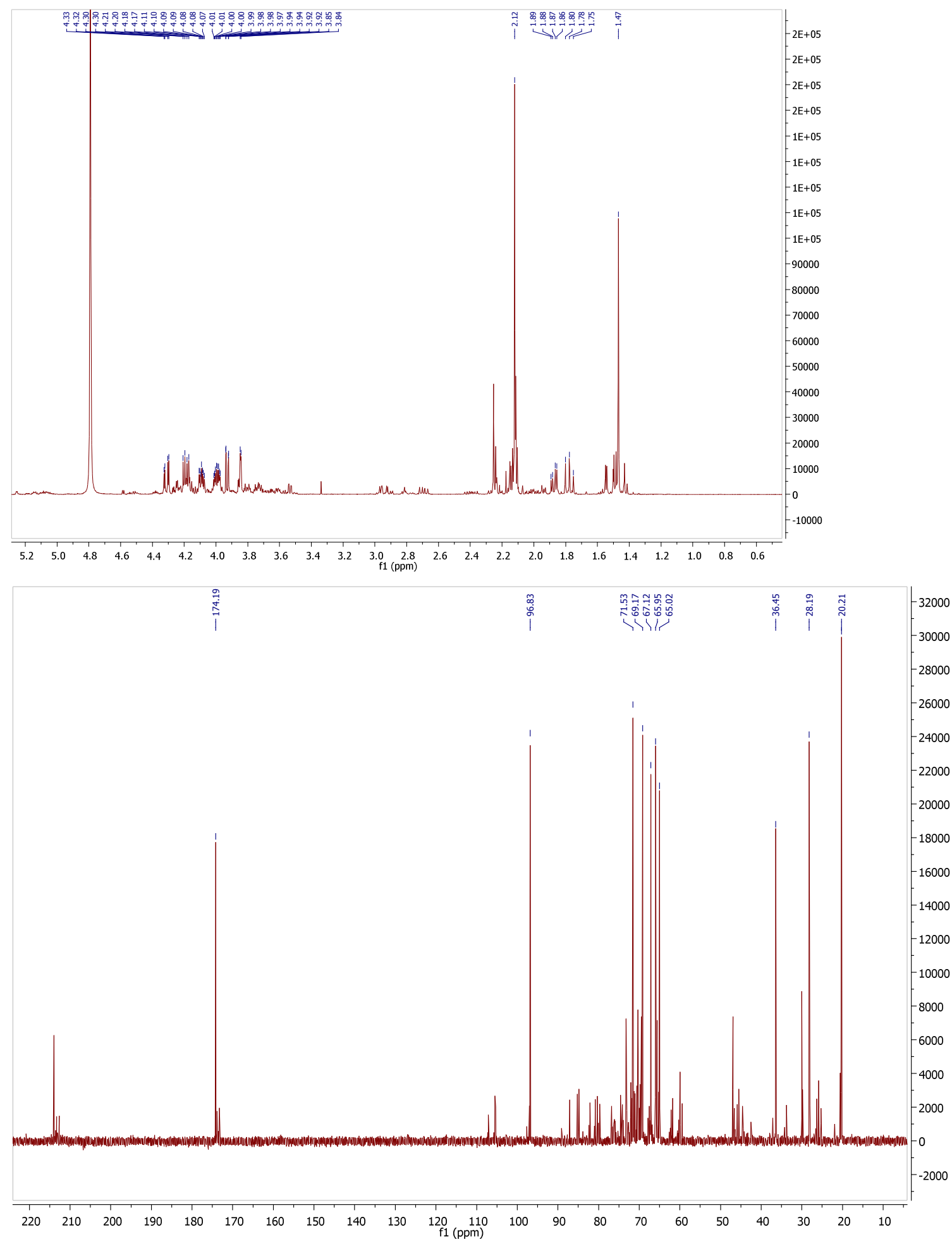


\section{(2R,3S,4S,5S)-7-oxooctane-1,2,3,4,5-pentayl pentaacetate anti-D-xylo-13}

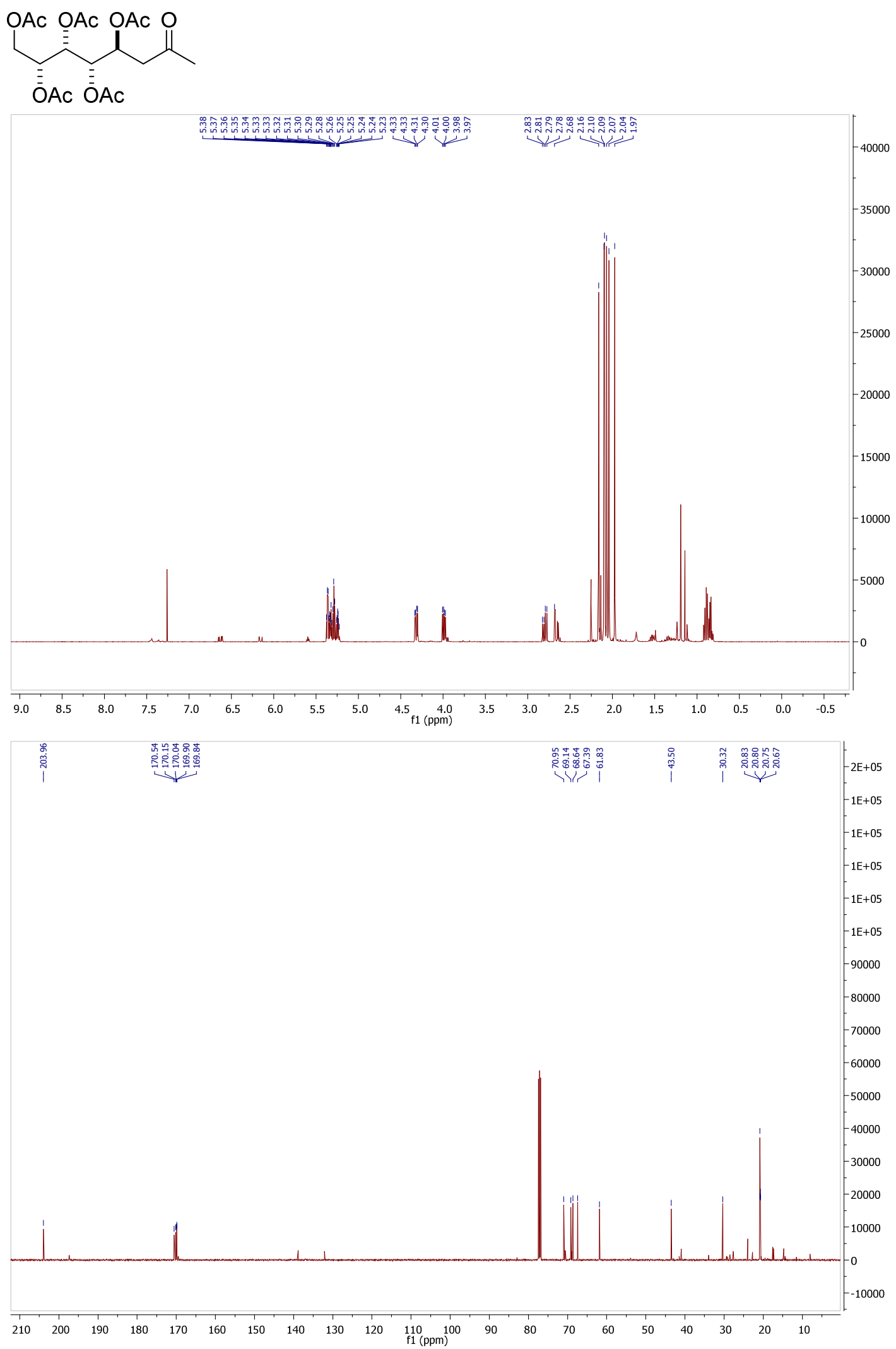




\section{(2R,3S,5S)-7-oxooctane-1,2,3,5-tetrayl tetraacetate D-deoxyribo-14}
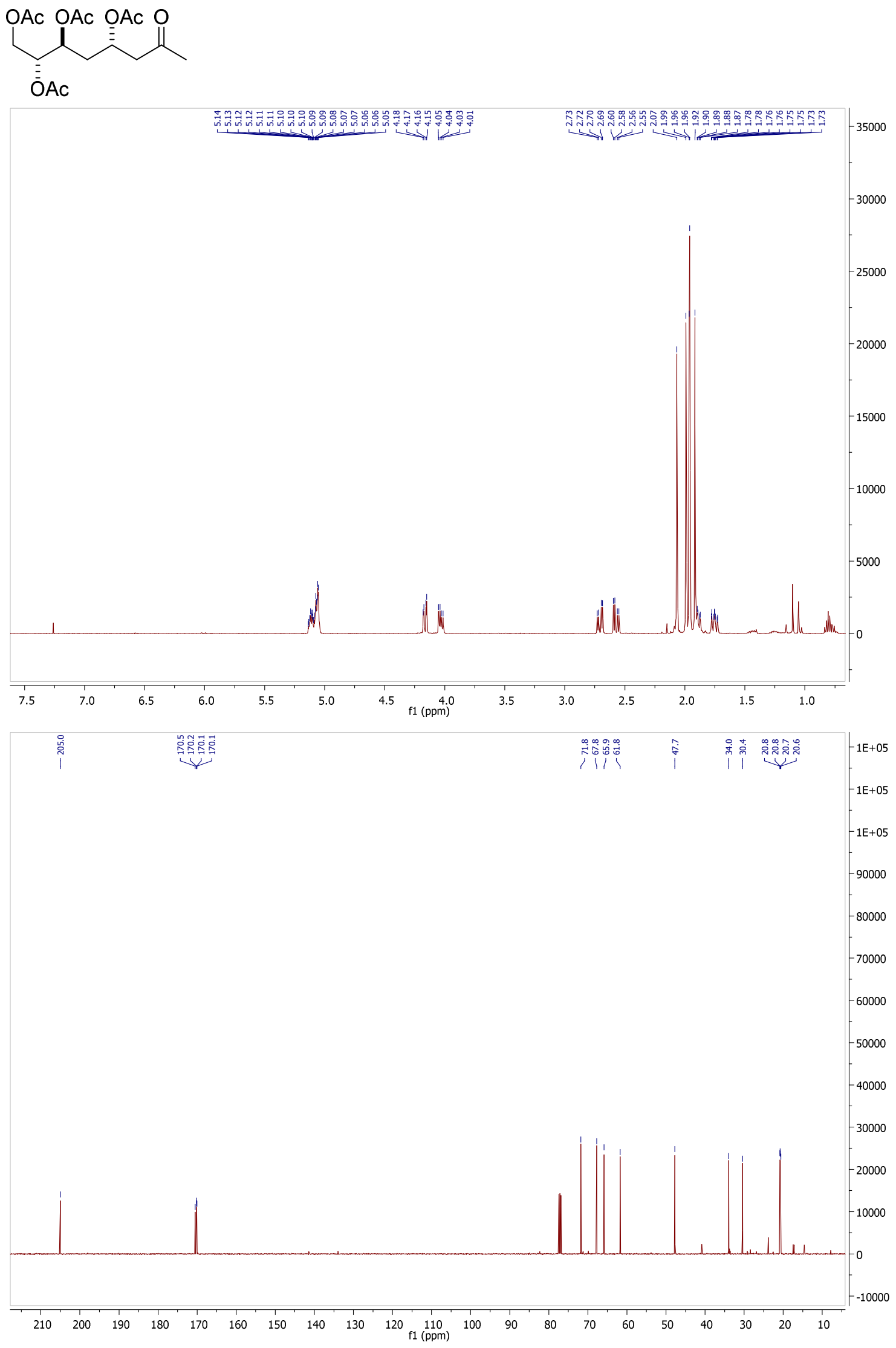
(2R,3R)-2,3-dihydroxy-3-((2R,3S,4S,6R)-3,4,6-trihydroxy-6-methyltetrahydro-2H-pyran-2-yl)propyl acetate<smiles>CC(=O)OC[C@H](O)C(O)C1(C(O)C(C)=O)O[C@](C)(O)C[C@H](O)[C@@H]1O</smiles>
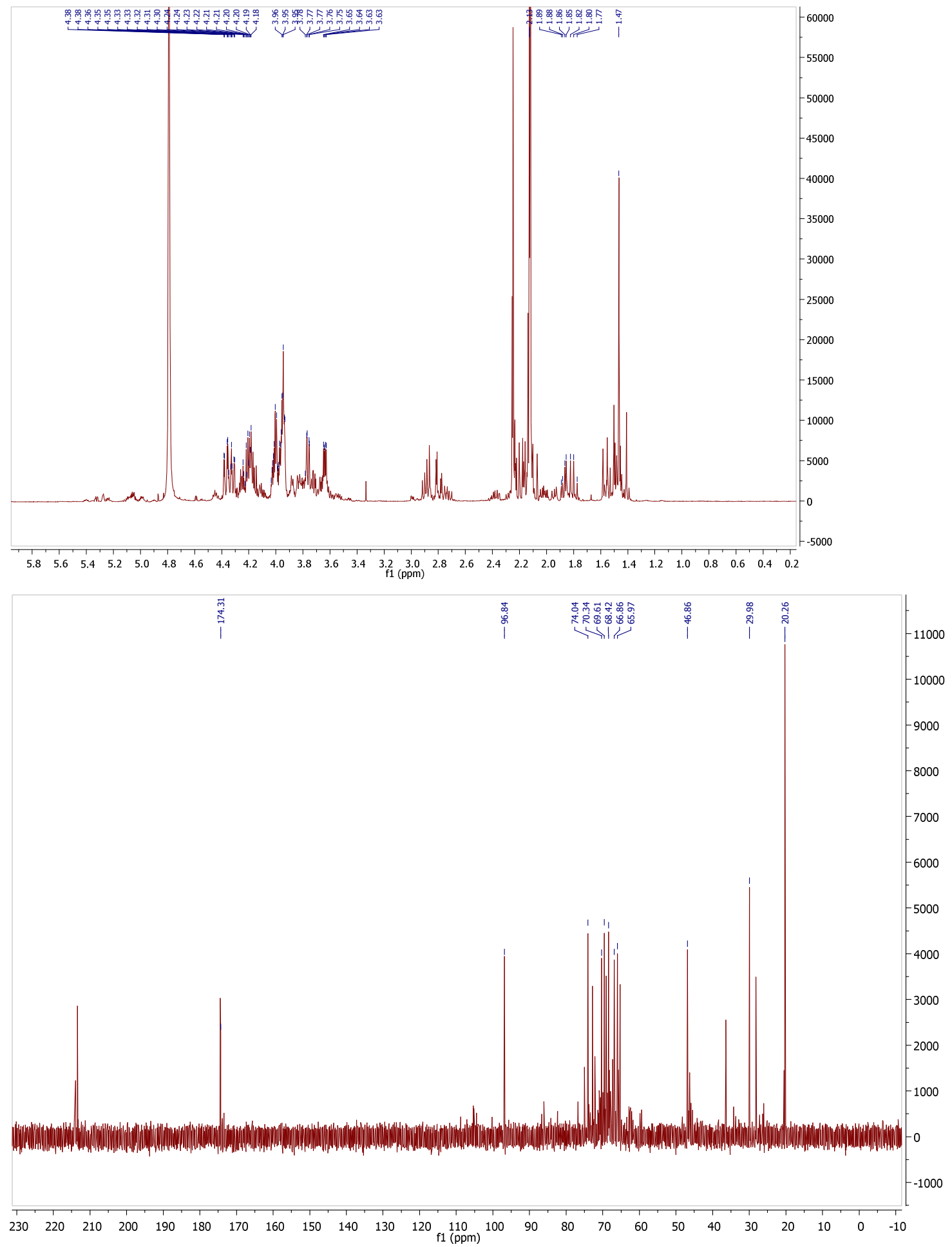
(2R,3R,4R,5S,6R)-8-oxononane-1,2,3,4,5,6-hexayl hexaacetate syn-D-gluco-20
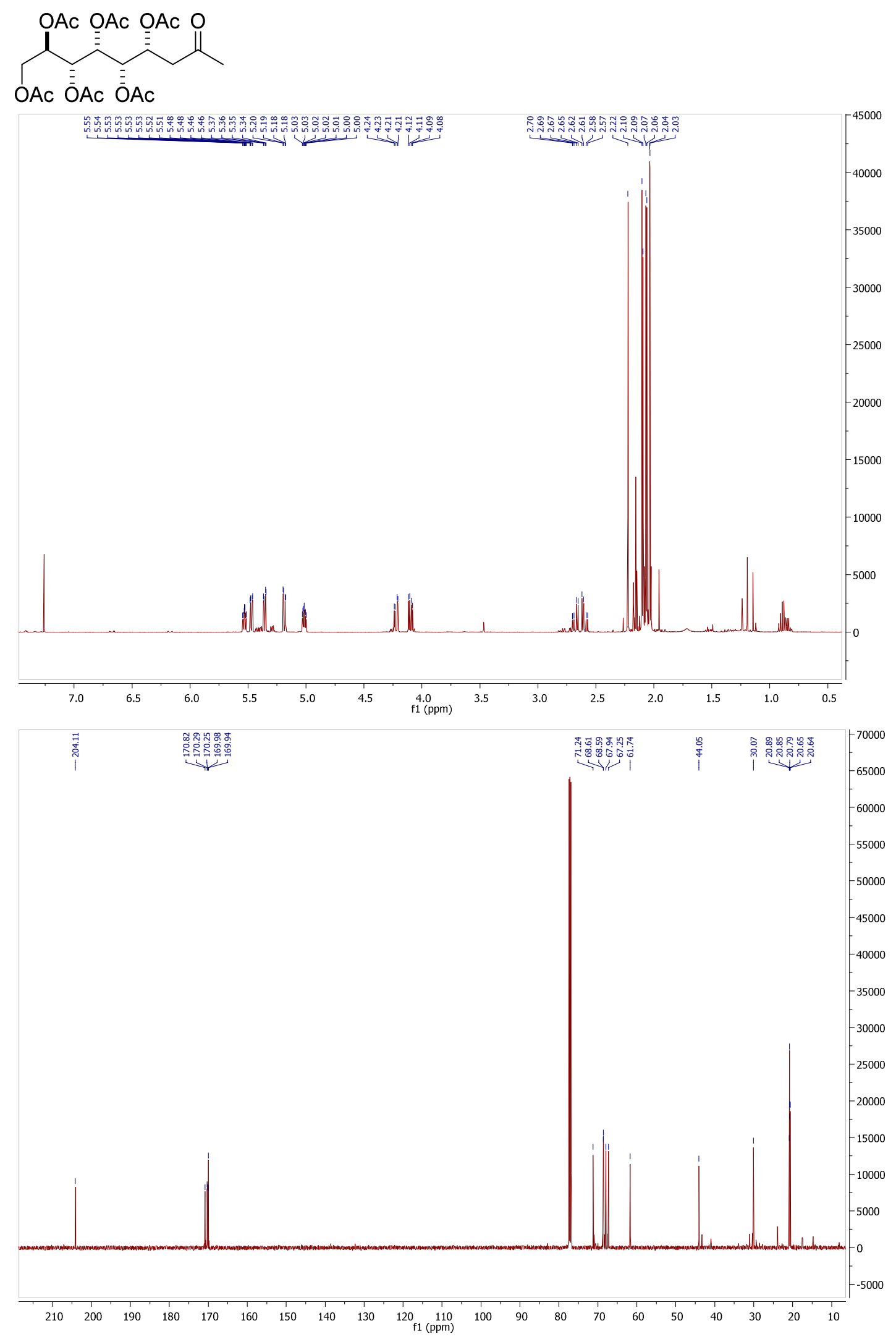
(2R,3R,4R,5S,6S)-8-oxononane-1,2,3,4,5,6-hexayl hexaacetate anti-D-gluco-20
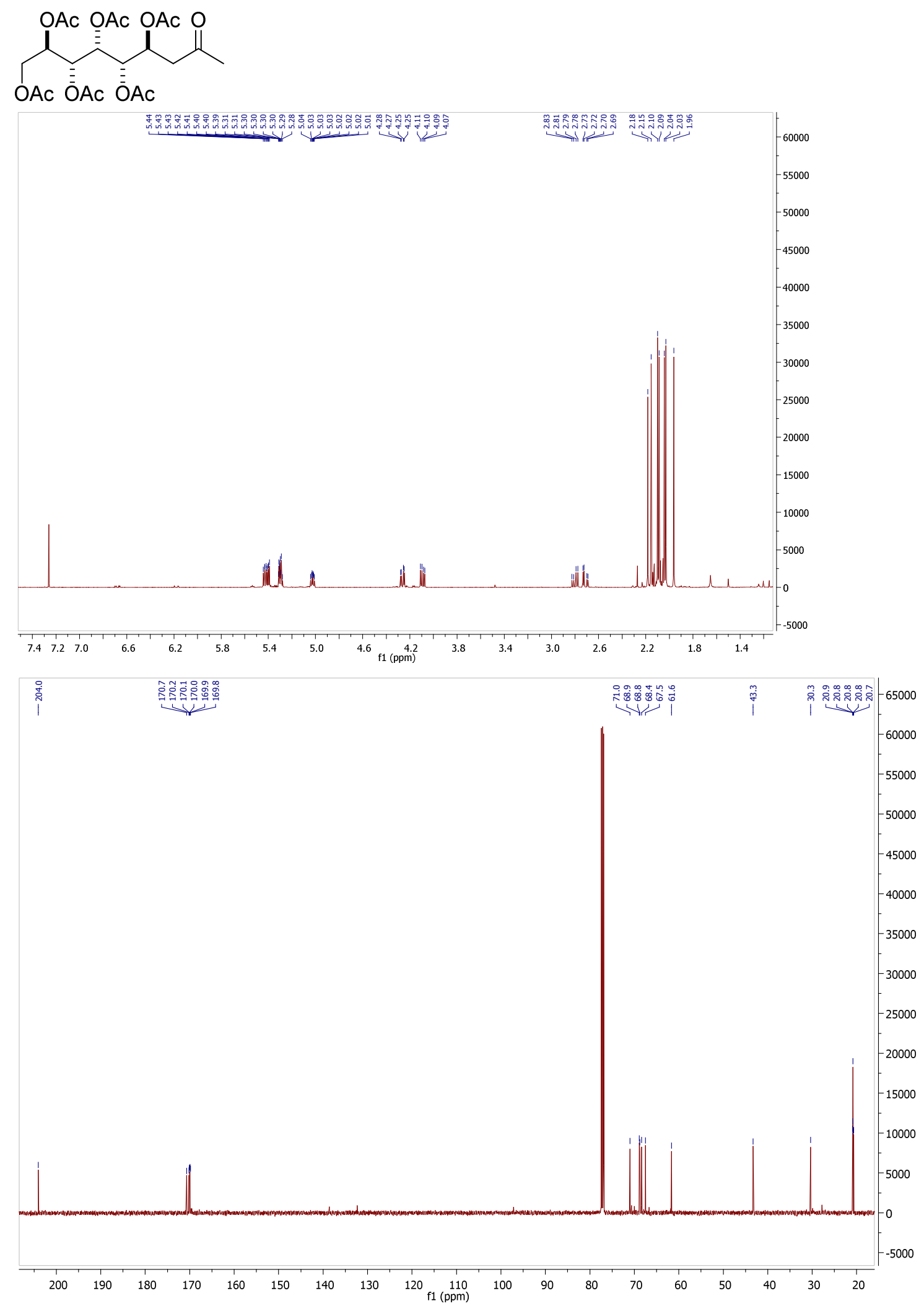
(2R,3S,4R,5S,6S)-8-oxononane-1,2,3,4,5,6-hexayl hexaacetate anti-D-galacto-21
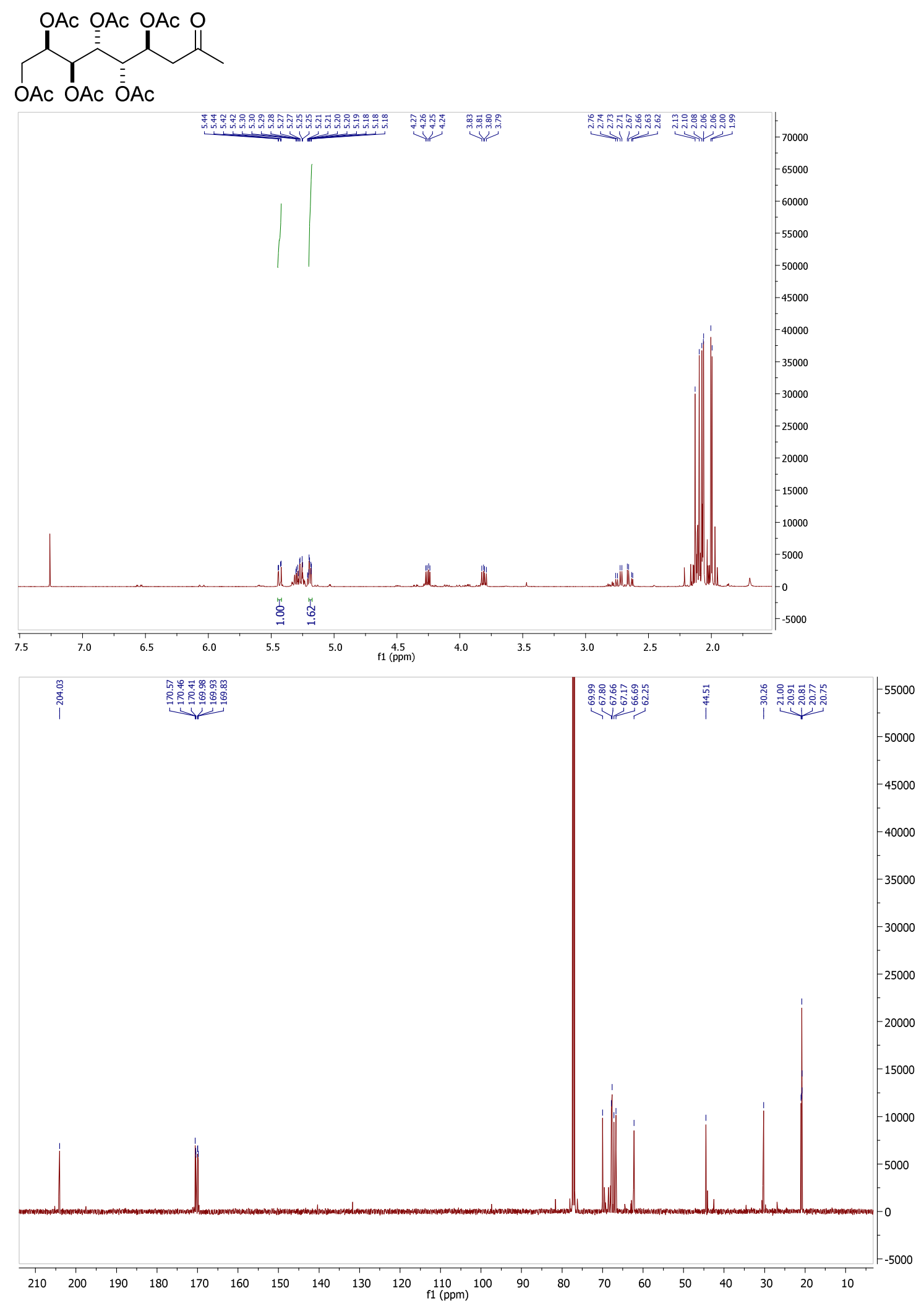


\section{(2R,3R,4R,5R,6R)-8-oxononane-1,2,3,4,5,6-hexayl hexaacetate anti-D-manno-22}
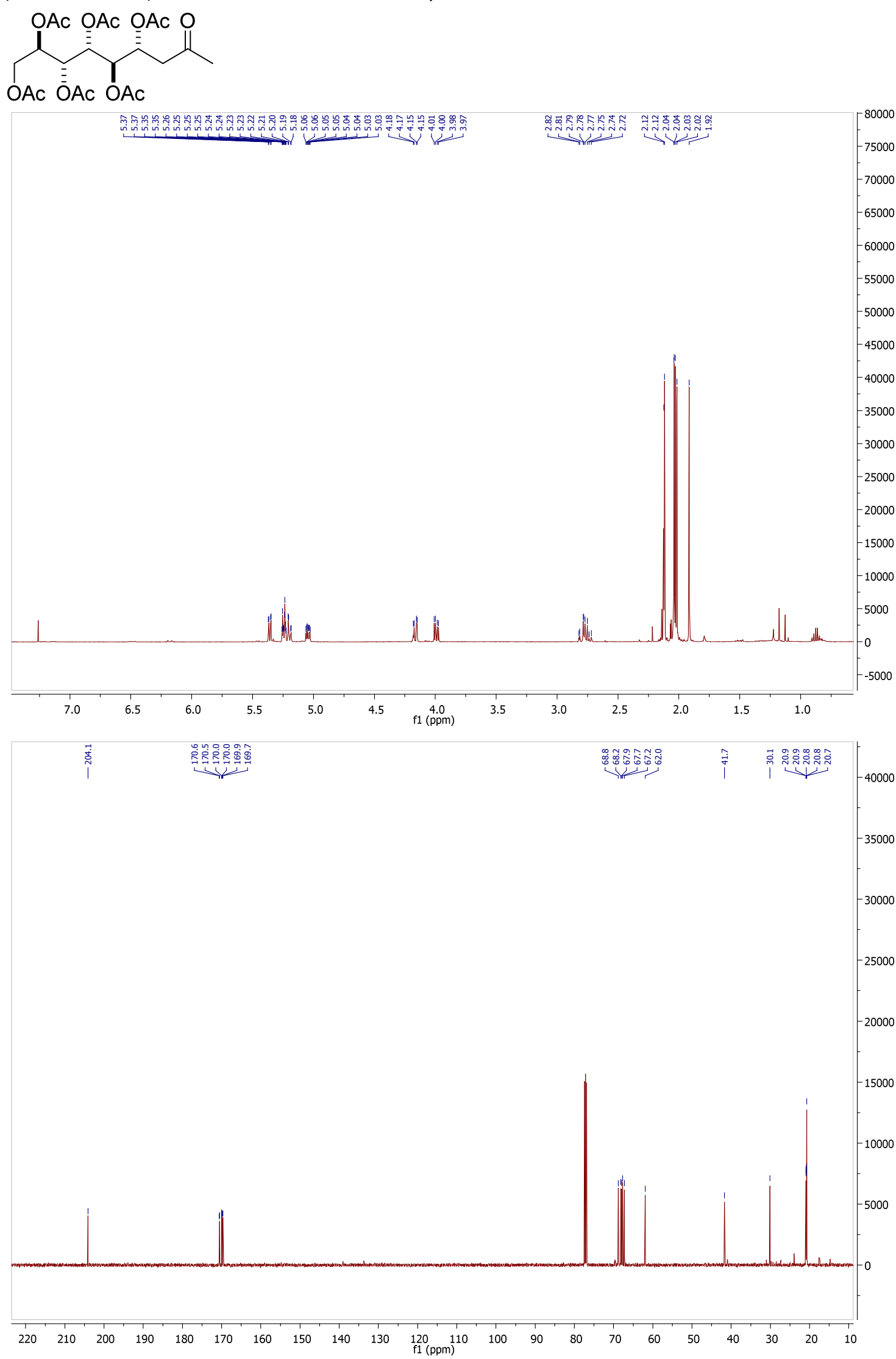
$(2 \mathrm{R}, 3 \mathrm{R}, 4 \mathrm{R}, 5 \mathrm{R}, 6 \mathrm{~S})-8$-oxononane-1,2,3,4,5,6-hexayl hexaacetate syn-D-manno-22 + (1R,2R)-1$((2 \mathrm{R}, 3 \mathrm{R}, 4 \mathrm{~S}, 6 \mathrm{R})-3,4$-diacetoxy-6-hydroxy-6-methyltetrahydro-2H-pyran-2-yl)propane-1,2,3-triyl triacetate syn-D-manno-22 (hemiketal)
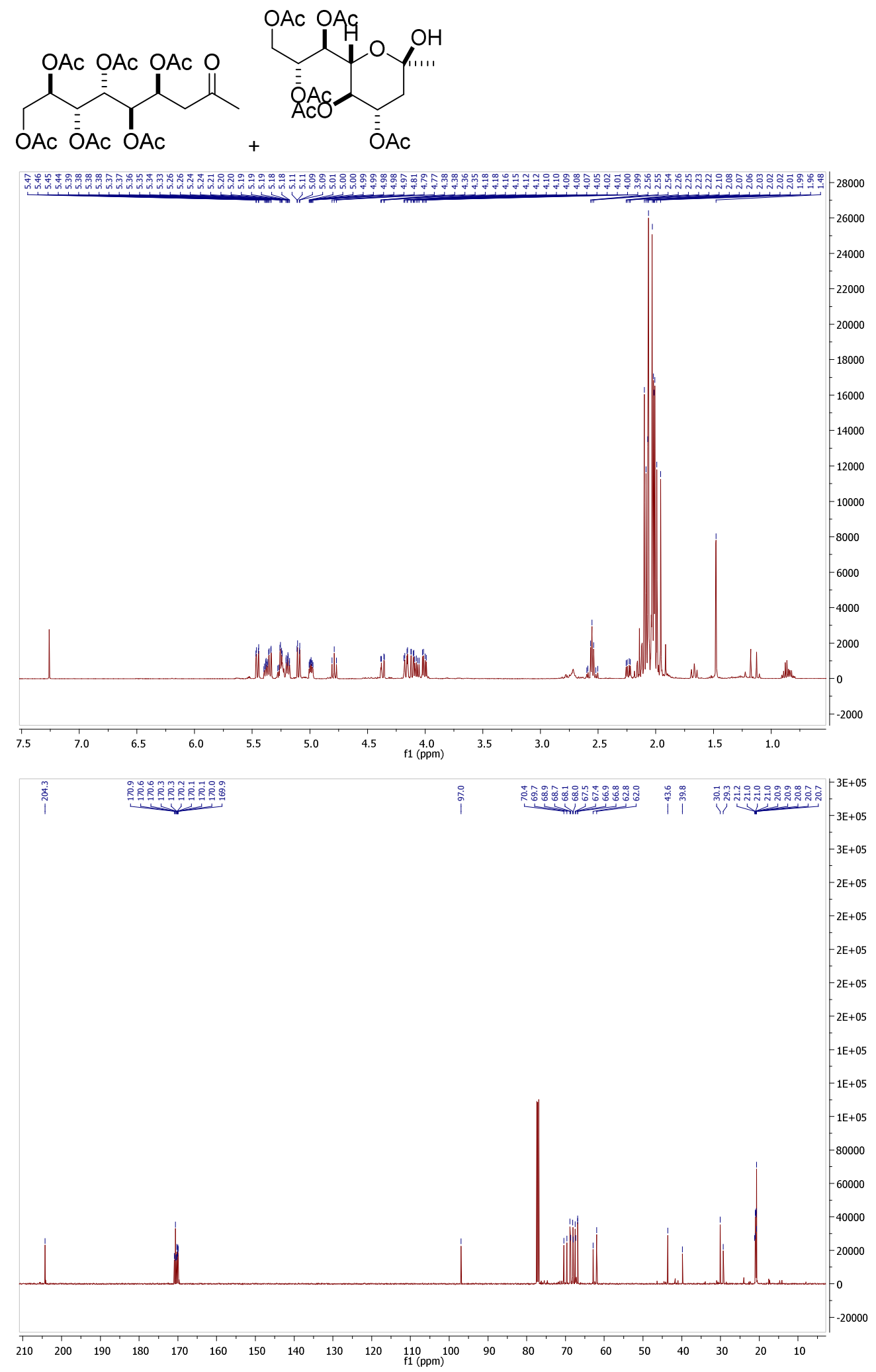
(2R,3S,4R,6R)-8-oxononane-1,2,3,4,6-pentayl pentaacetate D-deoxygluco-23
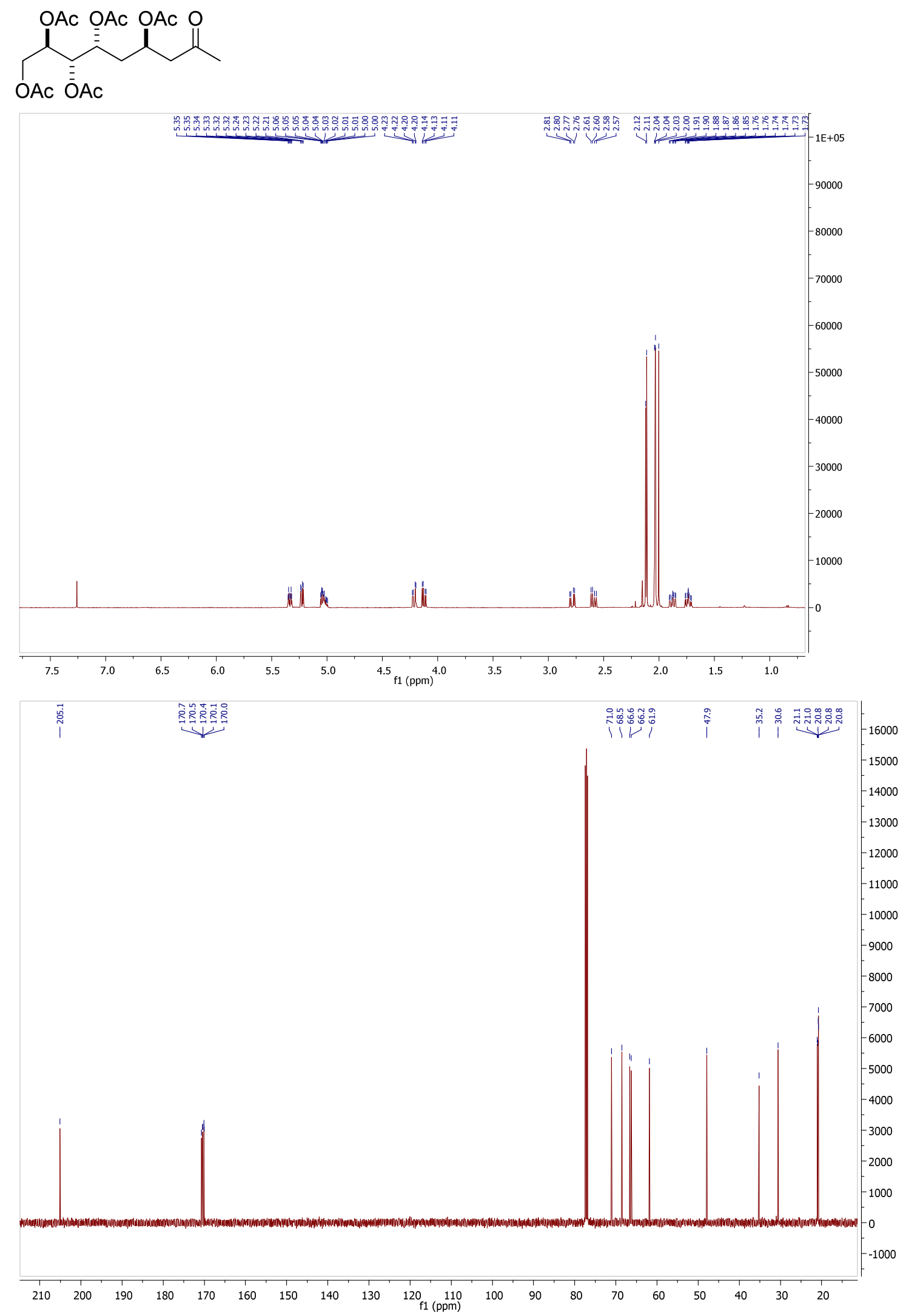


\section{(2R,3R,4R,6R)-8-oxononane-1,2,3,4,6-pentayl pentaacetate D-deoxygalacto-24}<smiles>CC(=O)CC(CC(CC(C)=O)C(OC(C)=O)C(C)=O)OC(C)=O</smiles>
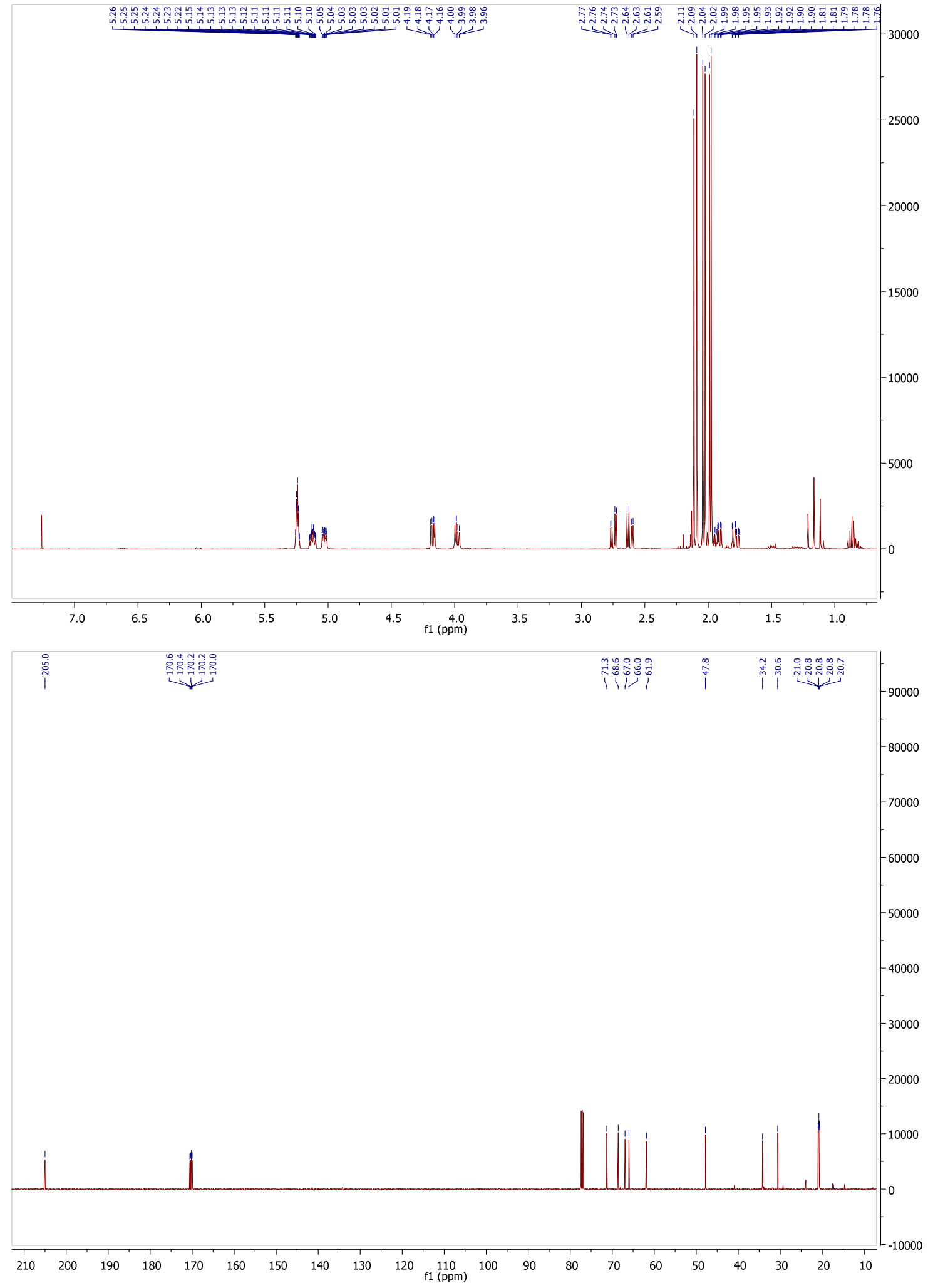
$(2 \mathrm{R}, 3 \mathrm{~S})-3-((2 \mathrm{R}, 4 \mathrm{R}, 5 \mathrm{~S}, 6 \mathrm{R})-4,6$-dihydroxy-5,6-dimethyltetrahydro-2H-pyran-2-yl)-2,3dihydroxypropyl acetate
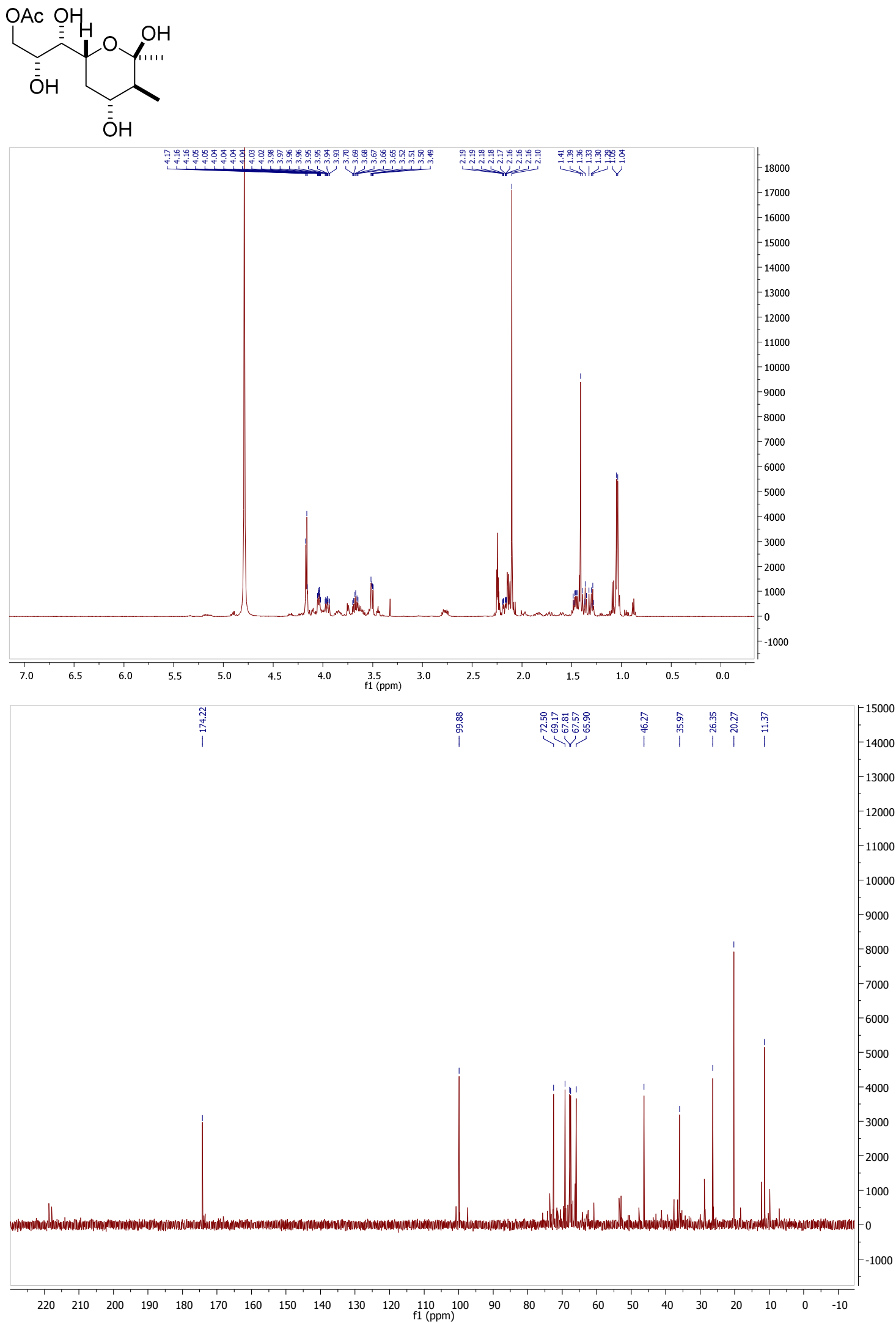


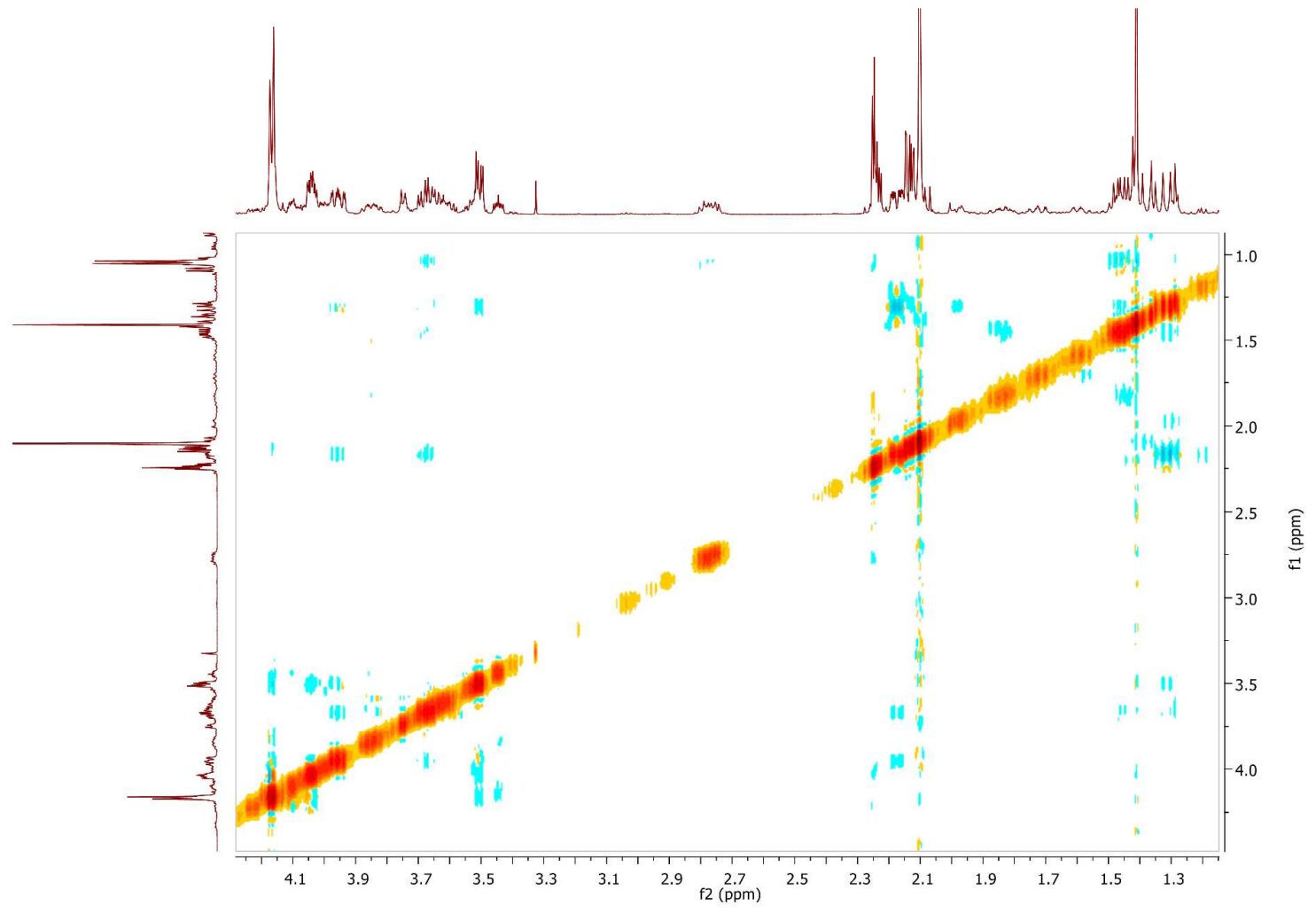


(2R,3R,4R,6R,7S)-7-methyl-8-oxononane-1,2,3,4,6-pentayl pentaacetate syn-25 + $\underline{(2 R, 3 R, 4 R, 6 R, 7 R)-7-m e t h y l-8-o x o n o n a n e-1,2,3,4,6-p e n t a y l ~ p e n t a a c e t a t e ~ a n t i-25 ~}$

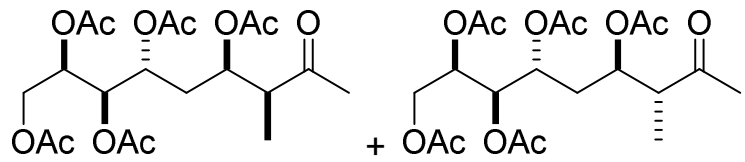
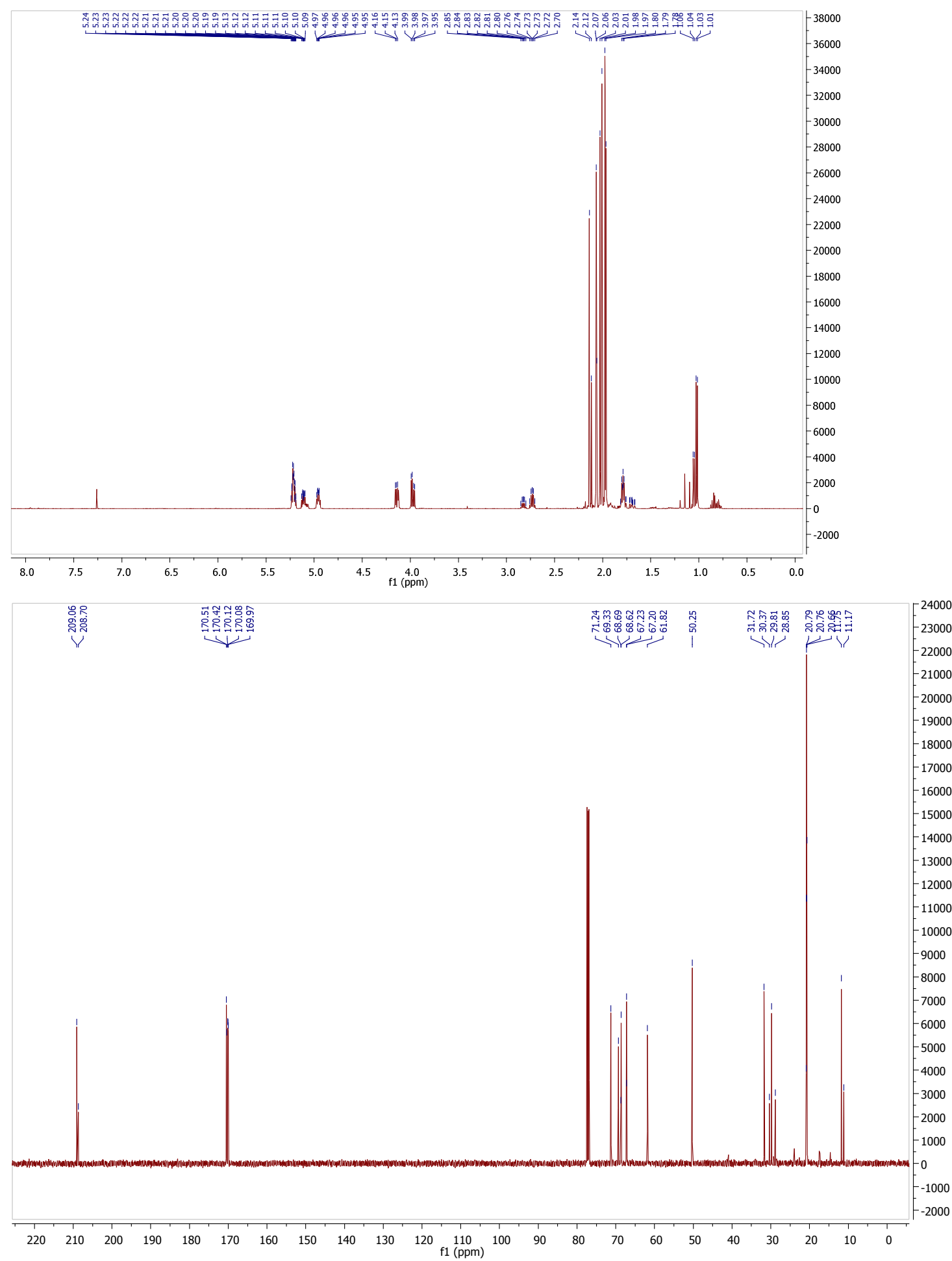